\title{
Determinants of menopause-related symptoms in women during the transition to menopause and the postmenopausal period - A systematic literature review
}

\author{
Zada Pajalić1* , Zora Raboteg-Šarić \\ 'Faculty of Health Sciences, VID Specialized University, Oslo, Norway, ${ }^{2}$ Institute of Social Sciences, Ivo Pilar, Zagreb, Croatia
}

\begin{abstract}
Introduction: Menopause is a complex physiological, self-perceived health condition caused by the reduction and termination of ovarian function, a process that results in a range of both psychological and physiological symptoms. The aim of this literature review was to synthesize the results of published studies on factors associated with menopausal symptoms in women during the transition to menopause and the postmenopausal period.
\end{abstract}

Methods: This systematic review was carried out according to the PRISMA statement. Primary studies published between 2007 and 2017 were identified through the following databases: PUBMED, SCOPUS, and CINAHL. In total, 3301 studies were identified and 279 were screened in full text. Of these, 54 studies were included for quality assessment using checklists from the Norwegian Institute for Public Health. A total of 22 studies were ultimately selected for inclusion in the results. These were thematically reviewed.

Results: A wide range of determinants was sorted as socio-demographic predictors, reproductive factors, clinical characteristics, behavioral, and lifestyle characteristics, and their association with the following outcomes was examined: The prevalence and severity of menopausal symptoms; sexual functioning; health indicators and health behaviors; health-related quality of life; physical performance; cognitive changes and attitudes; and the prevalence and age of natural menopause.

Conclusions: The current state of knowledge regarding menopause indicates that a wide array of instruments and variables have been employed and assessed to further understanding about menopause-related symptoms. This systematic review provides information on factors that should be considered in the treatment of menopause-related changes that may be bothersome for menopausal women.

Keywords: Determinants; menopause; psychological health; physical health; sexual health; symptoms

*Corresponding author: Zada Pajalić, Faculty of Health Sciences, VID
Specialized University, Oslo, Norway. E-mail: zada.pajalic@vid.no

Submitted: 06 February 2020/Accepted: 24 March 2020

\section{INTRODUCTION}

Menopause is a complex physiological process during which ovarian function and the production of sex hormones slowly diminish (1). Projections indicate that, by 2030, women aged 50 and older 
will number 1.2 billion; of these, $76 \%$ will be living in developing countries $(1,2)$. More than $50 \%$ of middle-aged women experience frequent menopause-related symptoms (3). These physiological changes result in a complex clinical picture and the experience of highly subjective symptoms. Because the symptoms are subjective, there is a risk that they may be underdiagnosed or undertreated (4). The increase of average life expectancy in the developed world is evident (5), and many social norms are under re-examination, such as the sphere of menopausal women's health and sexual health in older age. A national sample of 3005 adults between 57 and 85 years of age in the United States found that many older adults are sexually active (6), including women who experience an increase in menopause-related sexual difficulties (7-9).

The elderly population will expand between 2000 and 2050 (10). With an aging population, the number of women seeking help for menopause-related issues will increase (11). The menopausal transition is associated with sexual dysfunction for many women (12). Attitudes toward menopause range from negative to positive, and reflect the role of culture, attitude on the ageing process and the general life context of women (13).

Women's sexuality in welfare countries is in the midst of a transition away from gender norms, creating a new cultural scenario where women's sexual function after 40 is a way to see them as visible sexual beings (14). A study by Sayakhot et al. (2012) shows the differences in perceptions and experiences of menopause between women from Laos and Australia. Australian women more commonly reported menopause-related symptoms (psychological symptoms, sexual dysfunction, and vasomotor symptoms); similarities between groups included deficiencies in understanding with regard to menopausal therapies (15). Menopause is associated with a high risk of abnormalities of lipids and lipoproteins, insulin resistance and the development of cardiovascular diseases. Treatment with hormone replacement provides benefits against cardiovascular diseases but is dependent on the exact prescription, which is still being researched (16).

In a Brazilian population study, it was shown that $53.5 \%$ of menopausal women perceived their sex life as being good (17); in contrast, it was shown in a study from Spain that sexual satisfaction declines progressively after age 45 (18).

Menopause occurs, on average, at the age of $50(11,19,20)$, and results from a loss of ovarian function; as a result of this process, a woman's final menstruation marks that the period of fertility is over (provided there is no proven pathological condition) (2). Menopause is divided into perimenopause and postmenopause. Perimenopause is the result of a gradual loss of ovarian function over 5-10 years (19). Symptoms that occur during this period are menstruation irregularity, night sweats and hot flushes (21), genitourinary syndrome, and metabolic disorders; osteoporosis $(19,20)$. Chou et al.'s (2014) study in Macau, China showed that menopausal symptoms affect the quality of life in about $57.2 \%$ of women (22). Barlow et al. (1997) found that genitourinary syndrome caused by decreased production of estrogen affects about $48.8 \%$ of women (23), and manifests as itching, pain, dryness, vulvovaginal atrophy, and urinary retention.

Additional psychological symptoms that can occur are insomnia, nervousness, irritability, or depression. The period of menopause is characterized as a transition period during which there is often increased fear of diseases such as cancer, anxiety, poorer physiological health, and concern for obesity, and loss of attractiveness (19). Symptomatic vulvovaginal atrophy is common in approximately 50\% of postmenopausal women and can significantly impair their quality of life (24). A study by Hughes et al. (2015) showed that vaginal dryness as a sexual problem was typical for the majority of the study population (25). Another study confirmed these outcomes, also reporting that $67 \%$ of 49.5 -year-old women reported sexual dysfunction (26).

Menopause-related changes affect women's sexual health and may lead to sexual dysfunction (27). Yang et al. (2015) found that menopause-related physical changes can make sexual interaction difficult (28). Robak-Cholubek et al.'s (2014) study showed that degree of difficulty during the menopausal transition is associated with a level of adaptation to the changes - women who adapted to the changes had less frequently reported adverse 
experiences in their sexual interactions (29). A study by Cabral et al. (2014) showed that $94.5 \%$ of postmenopausal women respondents reported decreased sexual desire (30). A British National Survey confirmed that menopause-related symptoms were associated with low sexual function (31). In an Australian study that focused on the use of effective therapies for menopausal symptoms, it was shown that vaginal estrogen therapy is under-prescribed and under-treated (32). The occurrence and combination of different symptoms affect women's quality of life and are linked to their experience of health, sexuality, and the aging process. We developed our aims for this study in line with guidelines for literature reviews [27]. This systematic review aimed to synthesize the results of published studies on determinants associated with menopause-related symptoms in women during the transition to menopause and the postmenopausal period.

\section{METHODS}

This systematic review was carried out according to the PRISMA statement (33) and through use of Covidence, an online Cochrane technology platform for literature reviews (34). The search included studies published between 2007 and 2017. Only articles in English were included in the study. This review considered studies that included any outcome measure related directly or indirectly to menopause-related changes in women's health and women's experience of menopause. Inclusion criteria were primary studies that focused on the physiological menopause processes, and whose title and keywords included a type or types of menopause symptoms (e.g., "sexual dysfunction") or the terms "postmenopausal," "menopause," "women," "quality of life," "endocrine transition," and "menopausal transition." Peerreviewed original research studies were included in the study, as well as a range of study designs. Studies were included that enrolled women with natural or surgical menopause, used validated or non-validated tools, and were conducted in either community or hospital/clinic settings. Studies including men and women were included in the study, with a focus on results concerning menopausal women. Exclusion criteria consisted of studies that did not include the target population that did not constitute primary research; were review articles, books or book chapters that focused on diseases or infections (such as HIV or Sexually Transmitted Diseases), cancer, sexual minorities, mental health, pregnancy, tumors, incontinence, intimate partner violence, religiosity, blood pressure, systemic sclerosis, herpes, anxiety, distress, cytostatic treatment, hysterectomy, oophorectomy, pain, depression, partner violence, diabetes, premenstrual, youth, pregnancy, violence, or reproduction. Reports, abstracts, study protocols, conference proceedings, and case reports were excluded, as were reviews and studies employing a qualitative design. A systematic literature search was conducted in PUBMED, SCOPUS, and CINAHL in autumn 2017 to identify studies. The search strategy included the use of Medical Subject Headings terms related to each database. Table 1 shows an example of the search strategy used for the CINAHL database.

The records from the databases were imported into Endnote and then into XML formats and finally into Covidence (34). The review process had the following steps, all of which were conducted by both authors. Step one consisted of screening titles: The coauthors, one midwife, and one psychologist screened titles for relevance together using Covidence. In total, 3301 titles were included in the screening process. Both coauthors discussed each title and agreed on which titles to include and exclude. If there was disagreement on a particular article, the consensus was reached by discussion. Based on the inclusion/ exclusion criteria, 3021 titles were excluded from

TABLE 1. Example of search strategy in the CINAHL database

\begin{tabular}{llc}
\hline Search ID & Search terms & Results \\
\hline S1 & (MH "Climacteric") OR "climacteric" & 387 \\
S2 & (MH "Menopause") OR & 3.458 \\
& "MENOPAUSE" & \\
S3 & "Menopause treatment" & 4 \\
S4 & "Menopause symptoms" & 75 \\
S5 & (MH "Menopause") OR (MH "Women") & 9.496 \\
& OR "menopausal women" & \\
S6 & "Menopausia" & 13 \\
S7 & S1 AND S2 & 199 \\
S8 & S1 OR S2 & 3.458 \\
S9 & S1 OR S4 & 453 \\
S10 & S2 OR S5 & 10.609 \\
S11 & S2 AND S5 & 2.157 \\
S12 & S3 OR S4 & 78 \\
\hline
\end{tabular}


the study. In step two, the coauthors read abstracts of 279 titles separately. They then discussed these and agreed on the exclusion of 223 titles. In the final step, 54 full-text articles were read in their entirety, and quality assessment was achieved through the use of checklists for the evaluation of research from the Norwegian Institute for Public Health (35); a checklist for evaluation of cross-sectional studies based on the EBMH Notebook; guidelines for evaluating prevalence studies (36); and a checklist for cohort, longitudinal and RCT studies based on Guyatt et al. (2015) (37) and Singh (2013) (38). Finally, only papers that were assessed as being of medium or high quality were included in the study (Figure 1 and Tables 2-5).

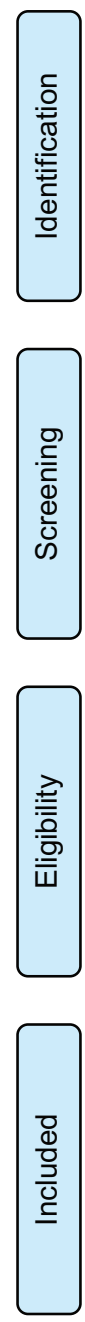

$$
(n=3301)
$$

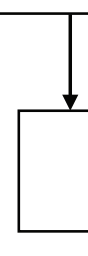

\section{Data extraction and synthesis}

Because of the marked heterogeneity of the included studies, all were narratively reviewed. The following data were extracted: General information (i.e., author, title, year, and country of origin); study characteristics (i.e., design and setting, and inclusion and exclusion criteria of the participants); characteristics of participants (number, age range, or mean age); assessment of exposure factors and covariates; assessment of outcomes; and main results. Studies based on data from larger follow-up studies were classified as cross-sectional if they included only data from a single follow-up assessment. Most studies included in this review were correlational, and in some cases, the outcome variables of interest - as well as all independent

\section{Additional records identified through other sources $(n=0)$}

Records after duplicates removed $(n=1)$
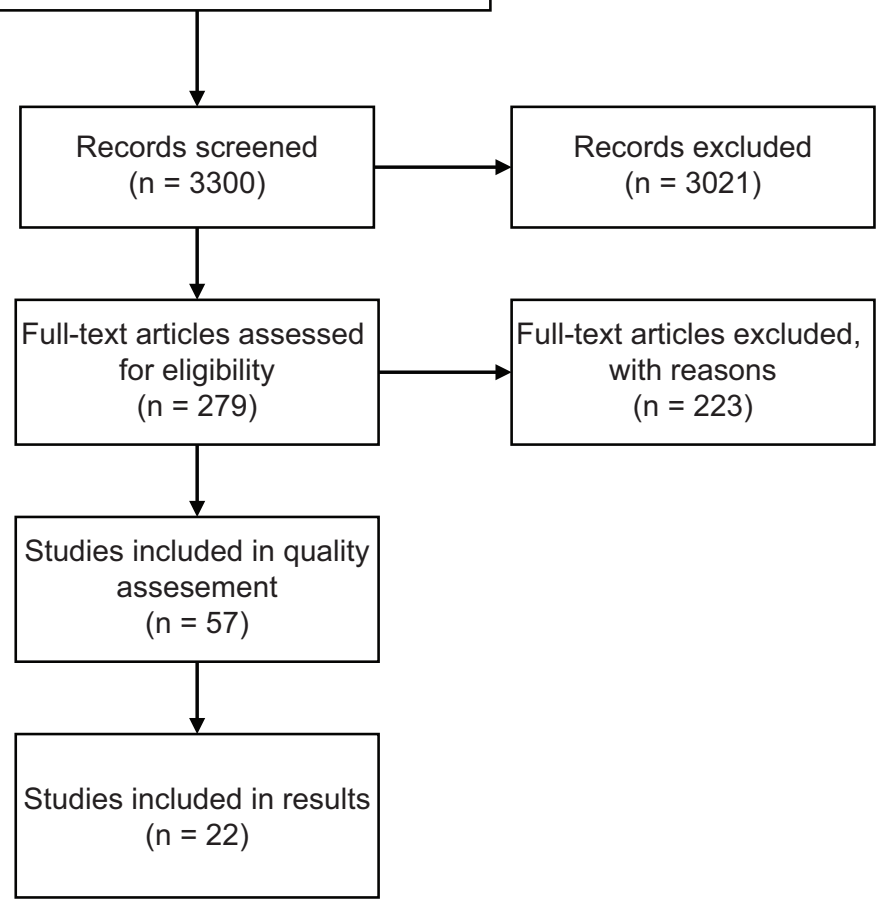

FIGURE 1. Flowchart of a selection of studies for the review. 
TABLE 2. Quality assessment of the included studies with a cross-sectional design

\begin{tabular}{|c|c|c|c|c|c|c|c|c|c|c|c|c|c|}
\hline Main author, year, country, title & 1 & 2 & 3 & 4 & 5 & 6 & 7 & 8 & 9 & 10 & 11 & 12 & $\begin{array}{l}\text { Total assessment } \\
\text { quality }\end{array}$ \\
\hline $\begin{array}{l}\text { Alquaiz et al., 2014, Saudi Arabia, Determinants of } \\
\text { the severity of menopausal symptoms among Saudi } \\
\text { women in Riyadh city }\end{array}$ & Y & Y & U & U & N & U & Y & Y & Y & Y & U & Y & Medium \\
\hline $\begin{array}{l}\text { Cabral et al., } 2013 \text {, Brasil, Determinants of sexual } \\
\text { dysfunction among middle-aged women }\end{array}$ & Y & Y & Y & Y & $\mathrm{N}$ & Y & Y & Y & Y & Y & U & Y & High quality \\
\hline $\begin{array}{l}\text { Da Câmara et al., 2015, Brasil, Menopausal status } \\
\text { and physical performance in middle-aged women: A } \\
\text { cross-sectional community-based study in Northeast } \\
\text { Brazil }\end{array}$ & Y & Y & Y & Y & $\mathrm{N}$ & Y & Y & Y & Y & Y & U & Y & High Quality \\
\hline $\begin{array}{l}\text { DiBonaventura, et al., 2015, US And Western } \\
\text { Europe, The association between Vulvovaginal } \\
\text { atrophy symptoms and quality of life among } \\
\text { postmenopausal women in the United States and } \\
\text { Western Europe }\end{array}$ & Y & Y & Y & U & $\mathrm{N}$ & U & Y & Y & Y & Y & Y & Y & Medium \\
\hline $\begin{array}{l}\text { Field et al., 2013, UK, Associations between health } \\
\text { and sexual lifestyles in Britain: Findings from the third } \\
\text { National Survey of Sexual Attitudes and Lifestyles } \\
\text { (Natsal-3) }\end{array}$ & Y & Y & Y & Y & Y & Y & Y & Y & Y & Y & Y & Y & High quality \\
\hline $\begin{array}{l}\text { Gartoulla et al., } 2015 \text {, Austria, Moderate to severe } \\
\text { vasomotor and sexual symptoms remain problematic } \\
\text { for women aged } 60-65 \mathrm{y}\end{array}$ & Y & Y & Y & Y & Y & Y & Y & Y & Y & Y & Y & Y & High quality \\
\hline $\begin{array}{l}\text { Ghazanfarpour et al., 2015, Iran, The relationship } \\
\text { between women's attitude towards menopause and } \\
\text { menopausal symptoms among postmenopausal } \\
\text { women }\end{array}$ & U & Y & Y & Y & N & U & Y & Y & Y & U & Y & Y & Medium \\
\hline $\begin{array}{l}\text { Hunter et al., 2016, US, Predictors of impact of } \\
\text { vaginal symptoms in postmenopausal women }\end{array}$ & Y & Y & Y & Y & Y & Y & Y & Y & Y & $Y$ & Y & Y & High quality \\
\hline $\begin{array}{l}\text { Jacobs et al., 2016, US, Impact of sex and } \\
\text { menopausal status on episodic memory circuitry in } \\
\text { early midlife }\end{array}$ & $Y$ & Y & Y & Y & U & Y & Y & Y & Y & Y & Y & Y & High quality \\
\hline $\begin{array}{l}\text { Jaspers et al., 2016, The Netherlands, Sex steroids, } \\
\text { sex hormone-binding globulin, and cardiovascular } \\
\text { health in men and postmenopausal women: The } \\
\text { Rotterdam study }\end{array}$ & $Y$ & Y & Y & Y & N & Y & Y & Y & Y & Y & U & Y & High quality \\
\hline $\begin{array}{l}\text { Javadivala et al., } 2013 \text {, Iran, Modeling the } \\
\text { relationship between physical activity and quality of } \\
\text { life in menopausal-aged women: A cross-sectional } \\
\text { study }\end{array}$ & $Y$ & Y & Y & U & N & U & Y & Y & Y & $U$ & Y & Y & Medium \\
\hline $\begin{array}{l}\text { Jonusiene et al., 2013, Lithuania, Sexual function, } \\
\text { mood, and menopause symptoms in Lithuanian } \\
\text { postmenopausal women }\end{array}$ & $Y$ & Y & U & U & Y & Y & Y & U & U & Y & Y & Y & Medium \\
\hline $\begin{array}{l}\text { Li et al., 2012, China, Factors associated with } \\
\text { the age of natural menopause and menopausal } \\
\text { symptoms in Chinese women }\end{array}$ & Y & Y & Y & Y & N & Y & U & Y & Y & $Y$ & Y & Y & High quality \\
\hline $\begin{array}{l}\text { Lo et al., 2015, China, Bone health status of } \\
\text { postmenopausal Chinese women }\end{array}$ & Y & Y & Y & $\mathrm{N}$ & $\mathrm{N}$ & U & Y & Y & Y & Y & Y & Y & High quality \\
\hline $\begin{array}{l}\text { Ornat et al., 2013, Spain, Sexual function, } \\
\text { satisfaction with life and menopausal symptoms in } \\
\text { middle-aged women }\end{array}$ & U & Y & Y & U & U & Y & Y & Y & Y & $Y$ & U & Y & Medium \\
\hline
\end{tabular}


TABLE 2. (Continued)

\begin{tabular}{llllllllllllll}
\hline Main author, year, country, title & 1 & 2 & 3 & 4 & 5 & 6 & 7 & 8 & 9 & 10 & 11 & 12 & $\begin{array}{l}\text { Total assessment } \\
\text { quality }\end{array}$ \\
\hline $\begin{array}{l}\text { Pallikadavath et al., 2016, India, Natural menopause } \\
\text { among women below } 50 \text { y in India: A population- }\end{array}$ & $\mathrm{Y}$ & $\mathrm{Y}$ & $\mathrm{Y}$ & $\mathrm{U}$ & $\mathrm{Y}$ & $\mathrm{U}$ & $\mathrm{Y}$ & $\mathrm{Y}$ & $\mathrm{Y}$ & $\mathrm{Y}$ & $\mathrm{U}$ & $\mathrm{Y}$ & Medium \\
based study & & & & & & & & & & & & & \\
$\begin{array}{l}\text { Prairie et al., 2015, US, Symptoms of depressed } \\
\text { mood, disturbed sleep, and sexual problems in } \\
\text { midlife women: Cross-sectional data from the study } \\
\text { of women's health across the nation }\end{array}$ & $\mathrm{Y}$ & $\mathrm{Y}$ & $\mathrm{Y}$ & $\mathrm{Y}$ & $\mathrm{U}$ & $\mathrm{Y}$ & $\mathrm{Y}$ & $\mathrm{Y}$ & $\mathrm{Y}$ & $\mathrm{Y}$ & $\mathrm{Y}$ & $\mathrm{Y}$ & High quality \\
$\begin{array}{l}\text { Som et al., 2014, India, Menopause-specific quality } \\
\text { of life of a group of urban women, West Bengal, India }\end{array}$ & $\mathrm{U}$ & $\mathrm{Y}$ & $\mathrm{Y}$ & $\mathrm{Y}$ & $\mathrm{N}$ & $\mathrm{U}$ & $\mathrm{U}$ & $\mathrm{Y}$ & $\mathrm{Y}$ & $\mathrm{Y}$ & $\mathrm{Y}$ & $\mathrm{Y}$ & Medium \\
$\begin{array}{l}\text { Yim et al., 2015, Korea, Prevalence and severity of } \\
\text { menopause symptoms and associated factors across }\end{array}$ & $\mathrm{Y}$ & $\mathrm{Y}$ & $\mathrm{Y}$ & $\mathrm{Y}$ & $\mathrm{N}$ & $\mathrm{Y}$ & $\mathrm{U}$ & $\mathrm{Y}$ & $\mathrm{Y}$ & $\mathrm{Y}$ & $\mathrm{Y}$ & $\mathrm{Y}$ & High quality \\
menopause status in Korean women
\end{tabular}

Checklist for cross-sectional studies based on Boyle MH: Guidelines for evaluating prevalence studies. Evidence-Based Mental Health 1998, 1(2):37-39. (1) Is the problem of the study formulated? (2) Is a prevalence study a suitable design to answer the issue/ research question? (3) Is the population from which the sample is taken clearly defined? (4) Was the selection included in the study in a satisfactory manner? (5) Has it been clarified whether the respondents differ from those who have not responded? (6) Is the response rate high enough? (7) Does the study use measurement methods that are reliable (valid) for what you want to measure? (8) Is data collection standardized? 9) Is data analysis standardized? (10) What is the result of this study? (11) Can the results be transferred to practice? (12) Do the results of this study fit with the results of other available studies? (Answers Yes: Y; No: N; Unclear: U)

TABLE 3. Overview criteria met and the quality of the study

\begin{tabular}{ll}
\hline Criteria met & The quality of the study \\
\hline All/almost all criteria met. Any weaknesses cannot change the conclusion of the study & High quality \\
Used if any of the criteria from the checklist are not met or if the criteria are not satisfactorily described. & Medium quality \\
It is assumed, however, that there is little chance that the weaknesses could have changed the study's & \\
conclusion & \\
$\begin{array}{l}\text { They are used if few or no criteria from the checklist are met or are not satisfactorily described. The } \\
\text { weaknesses may mean that the conclusion of the study is wrong }\end{array}$ & Inadequate/low \\
No criteria met & Excluded \\
\hline
\end{tabular}

variables and covariates - were not clearly stated in the text of the article. In such cases, variables were classified by carefully examining the study aims, data analyses, and tables in the article's results section.

The main results concerning the determinants and consequences of menopause-related symptoms in women are summarized in Table 6 . The number of participants for a specific study presented in Table 6 corresponds to the number of participants selected for analysis based on the inclusion/exclusion criteria and completed questionnaires (in some articles, the number of participants presented in the abstract includes the initial sample). As the included studies collected data for a broad range of outcomes, descriptive synthesis was organized according to similar outcomes and factors significantly associated with those outcomes.
The primary outcomes of interest in this review were: (1) Menopausal symptoms (39-47); (2) sexual functioning $(43,44,48-52)$; (3) health indicators and health behaviors $(41,53-55)$; and (4) health-related quality of life $(41,43,52,56)$. Secondary outcomes of interest included physical performance and cognitive functioning $(57,58)$; attitudes toward menopause $(45,59)$; and the age and prevalence of natural menopause $(42,60)$.

The classification of exposure or independent variables and potential confounding variables in the included 22 studies is presented in Table 7 . All studies examined the effects of women's age as a biological and socio-demographic characteristic. Other frequently examined demographic characteristics were educational level (18 studies); marital/relationship 
TABLE 4. Quality assessment of the included studies with cohorts design

\begin{tabular}{lllllllllllllll}
\hline Main author, year, country, title & 1 & 2 & 3 & 4 & 5 & 6 & 7 & 8 & 9 & 10 & 11 & 12 & Total assessment quality \\
\hline $\begin{array}{l}\text { Kutob et al., 2017, US, Relationship between marital } \\
\text { transitions, health behaviors, and health indicators of }\end{array}$ & $\mathrm{Y}$ & $\mathrm{U}$ & $\mathrm{Y}$ & $\mathrm{Y}$ & $\mathrm{Y}$ & $\mathrm{Y}$ & $\mathrm{U}$ & $\mathrm{Y}$ & $\mathrm{Y}$ & $\mathrm{Y}$ & $\mathrm{Y}$ & $\mathrm{Y}$ & High quality \\
postmenopausal women: Results from the women's health \\
initiative
\end{tabular}

Checklist for cohorts studies (1) Is the purpose of the study formulated? (2) Were subjects recruited for the cohort satisfactorily?

(3) Was the exposure accurately measured? (4) Was the outcome accurately measured? (5) A. Have the authors identified all important confounders in the study's design and analysis? (5) B. Have the authors take into account known, possible confounders in the design and analysis of the study? (6) Were any of the people in the cohort followed up? (7) Were the people followed up long enough? 8) What is the result of this study? (9) Do you trust the results? (10) Can the results be transferred to practice? (11) Do the results of this study fit with the results of other available studies? (12) What are the implications of this study for practice? (answers Yes: Y; No: N; Unclear: U)

1. Guyatt G, Rennie D, Meade M, Cook D. Users' guides to the medical literature: McGraw-Hill Medical:; 2015

2. Magne Nylenna: Slik oppsummerer v forskning. Håndbok for Nasjonalt kunnskapssenter for helsetjenesten. vol. 4: Oslo: Nasjonalt kunnskapssenter for helsetjenesten; 2015; 2006

3. Singh J: Critical appraisal skills programme. Journal of Pharmacology and Pharmacotherapeutics 2013, 4(1):76

TABLE 5. Quality assessment of the included studies with an RCT design

\begin{tabular}{lllllllllllll}
\hline Main author, year, country, title & 1 & 2 & 3 & 4 & 5 & 6 & 7 & 8 & 9 & 10 & 11 & $\begin{array}{l}\text { Total assessment } \\
\text { quality }\end{array}$ \\
\hline $\begin{array}{l}\text { Tungmunsakulchai et al., 2015 Thailand, The effectiveness of a low } \\
\text { dose of testosterone undecanoate to improve sexual function in } \\
\text { postmenopausal women }\end{array}$ & $\mathrm{U}$ & $\mathrm{Y}$ & $\mathrm{Y}$ & $\mathrm{Y}$ & $\mathrm{Y}$ & $\mathrm{Y}$ & $\mathrm{Y}$ & $\mathrm{Y}$ & $\mathrm{U}$ & $\mathrm{Y}$ & $\mathrm{Y}$ & High quality \\
\hline
\end{tabular}

Checklist for RCT studies: (1) Is the purpose of the study clearly formulated? (2) Was the assignment to the different groups using a satisfactory randomization procedure? (3) Were all the participants reported at the end of the study? (4) Were participants, health-care providers, and study personnel "blind" to treatment? (5) Were the groups similar at the start of the trial? (6) Aside from the measure being evaluated (experimental intervention), were the groups treated equally? (7) What are the results? (8) How accurate are the results? (9) Can the results be transferred to practice? 10). Were all important outcomes measured in this study? (11) Are the benefits worth the harms and costs? (answers: Yes: Y; No: N; Unclear: U)

status (16 studies); and indicators of socioeconomic status, such as family income (12 studies) and women's occupation or employment (seven studies). Among the independent reproductive variables, the most frequently examined were women's reproductive stage (14 studies) and parity (eight studies). Body mass index (BMI) was the most frequently examined independent variable among clinical characteristics (14 studies), while smoking (ten studies) and physical activity or exercise (ten studies) were the most frequently examined lifestyle and behavioral characteristics.

\section{Description of eligible studies}

The eligible studies were published between 2012 and 2017. The studies ranged in sample size from 70 to 630,361 women, due to different methodologies and study aims. Two studies - one randomized controlled trial (51) and one functional magnetic resonance study (57) - included 100 women or fewer. In nine studies, the number of participants ranged between 246 and $389(39,41,43,45,46,48,50,58,59)$, while five studies included 745-1774 women $(44,47,52,53,55)$, and six studies enrolled more than 2020 women $(40,42,49,54,56,60)$. Of these, three studies had more than 20,000 participants. Nineteen of the included studies were cross-sectional $(39,44,46-50,53,55-60)$, two studies were longitudinal $(45,54)$, and one study used a randomized controlled trial design (51).

Almost all studies were single-country studies and were conducted in 14 countries worldwide: 


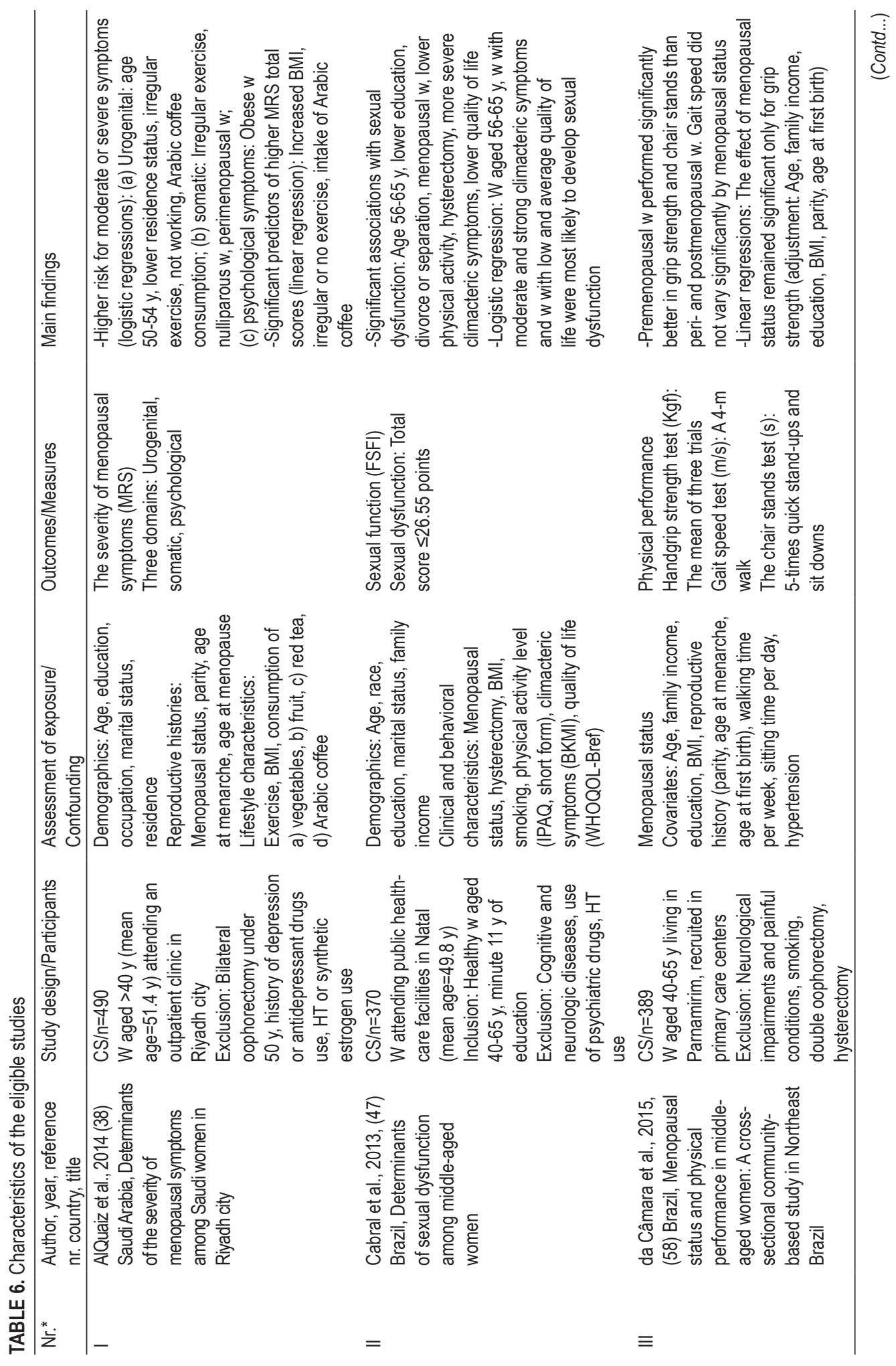




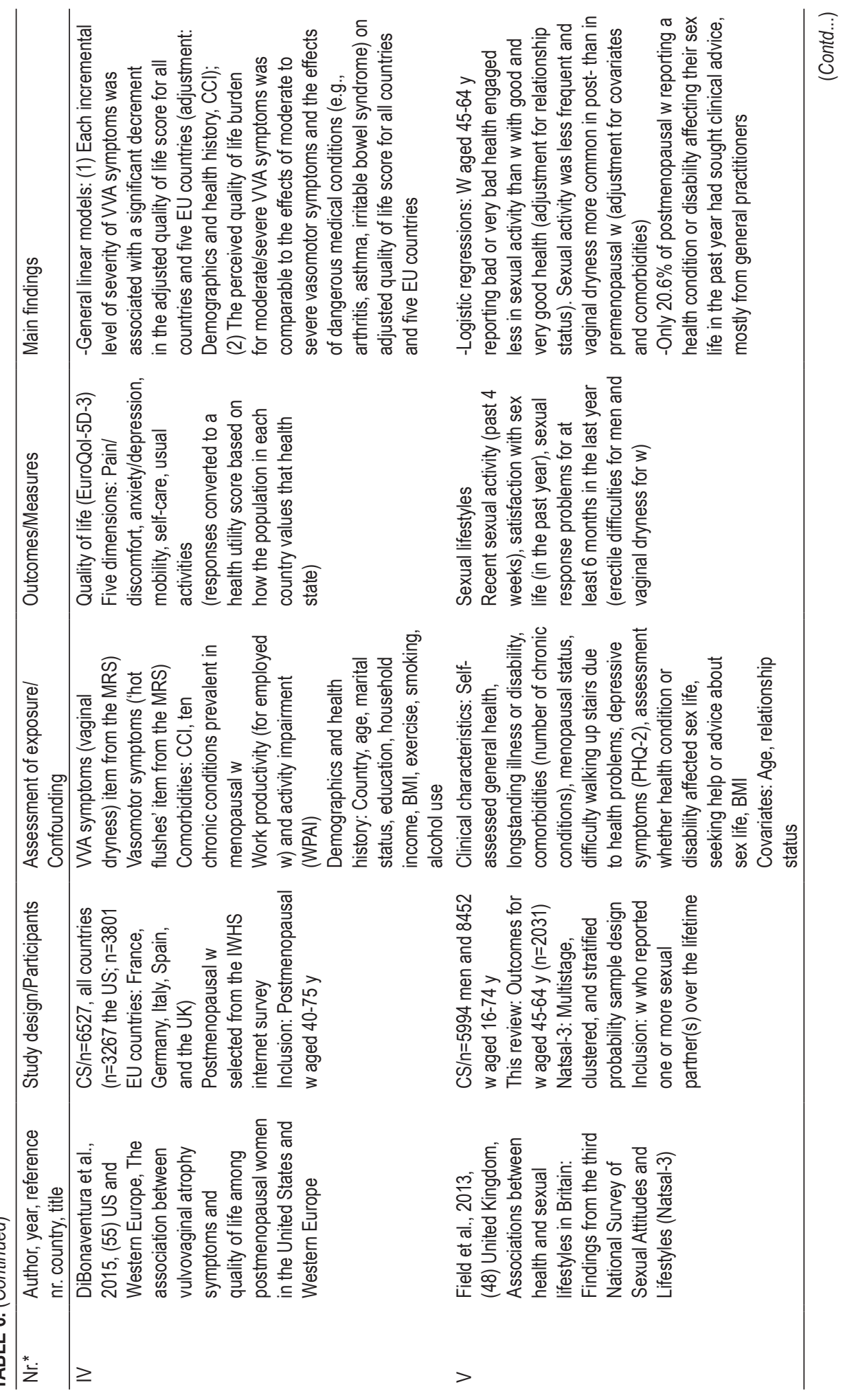




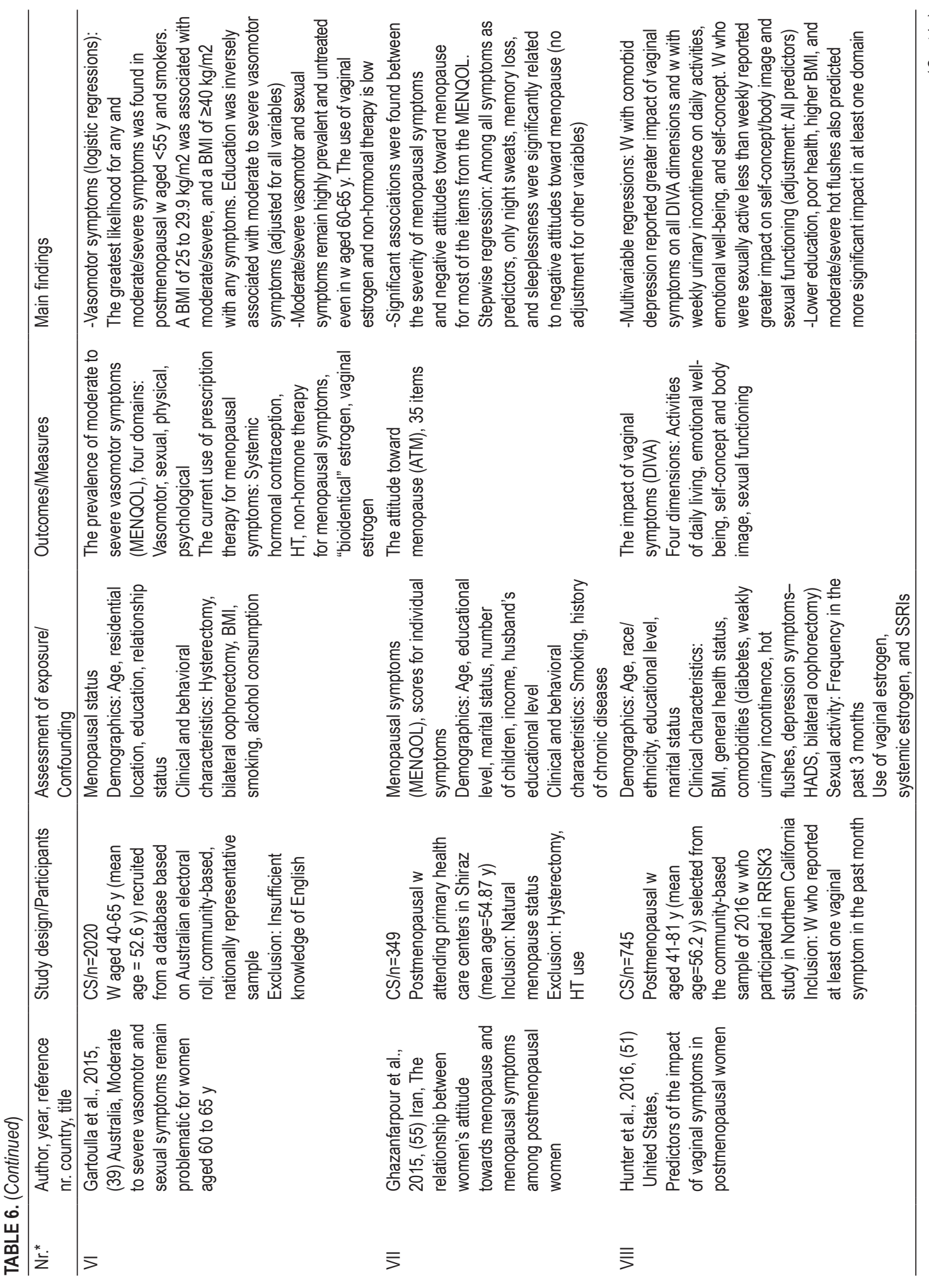




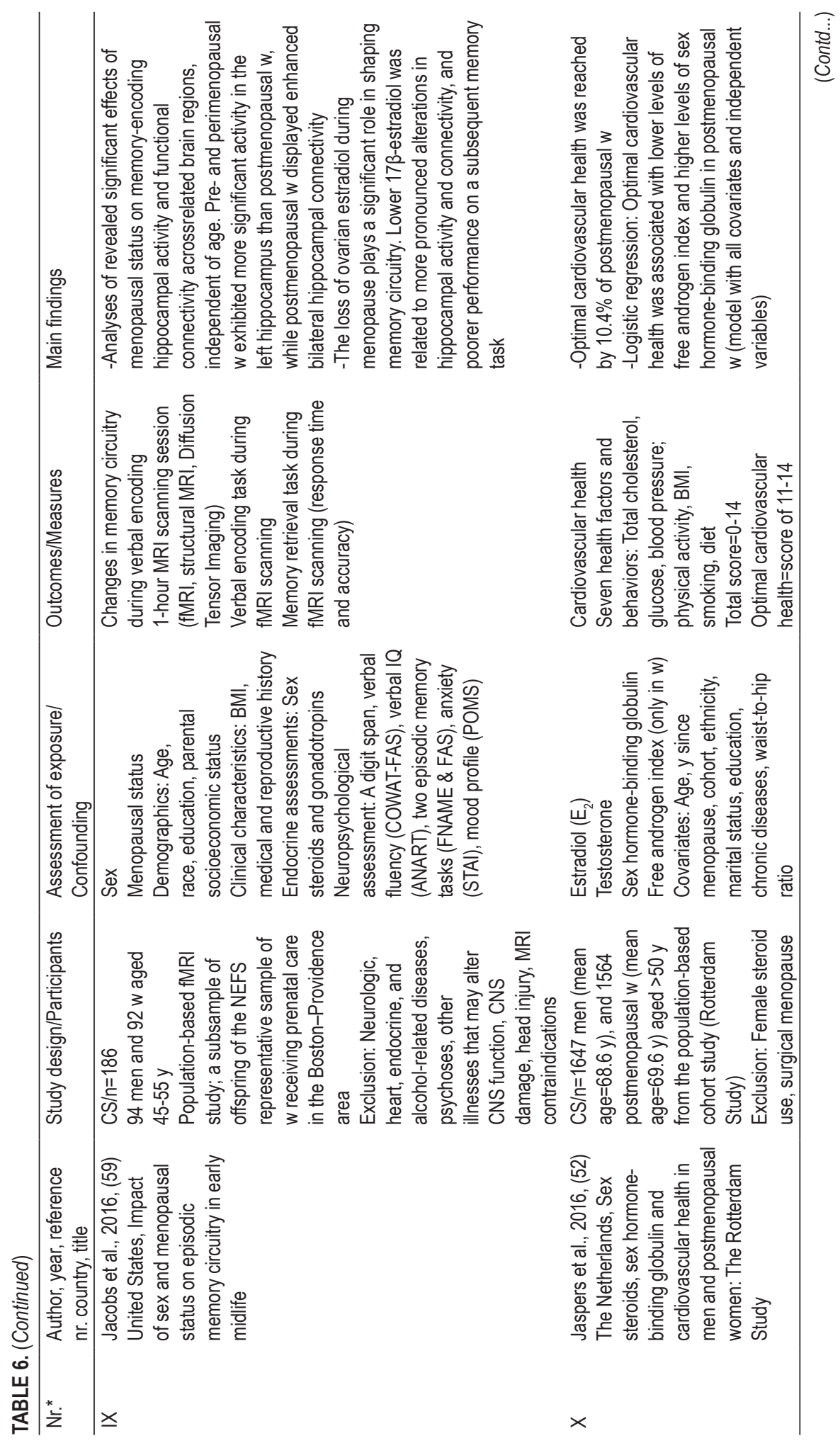




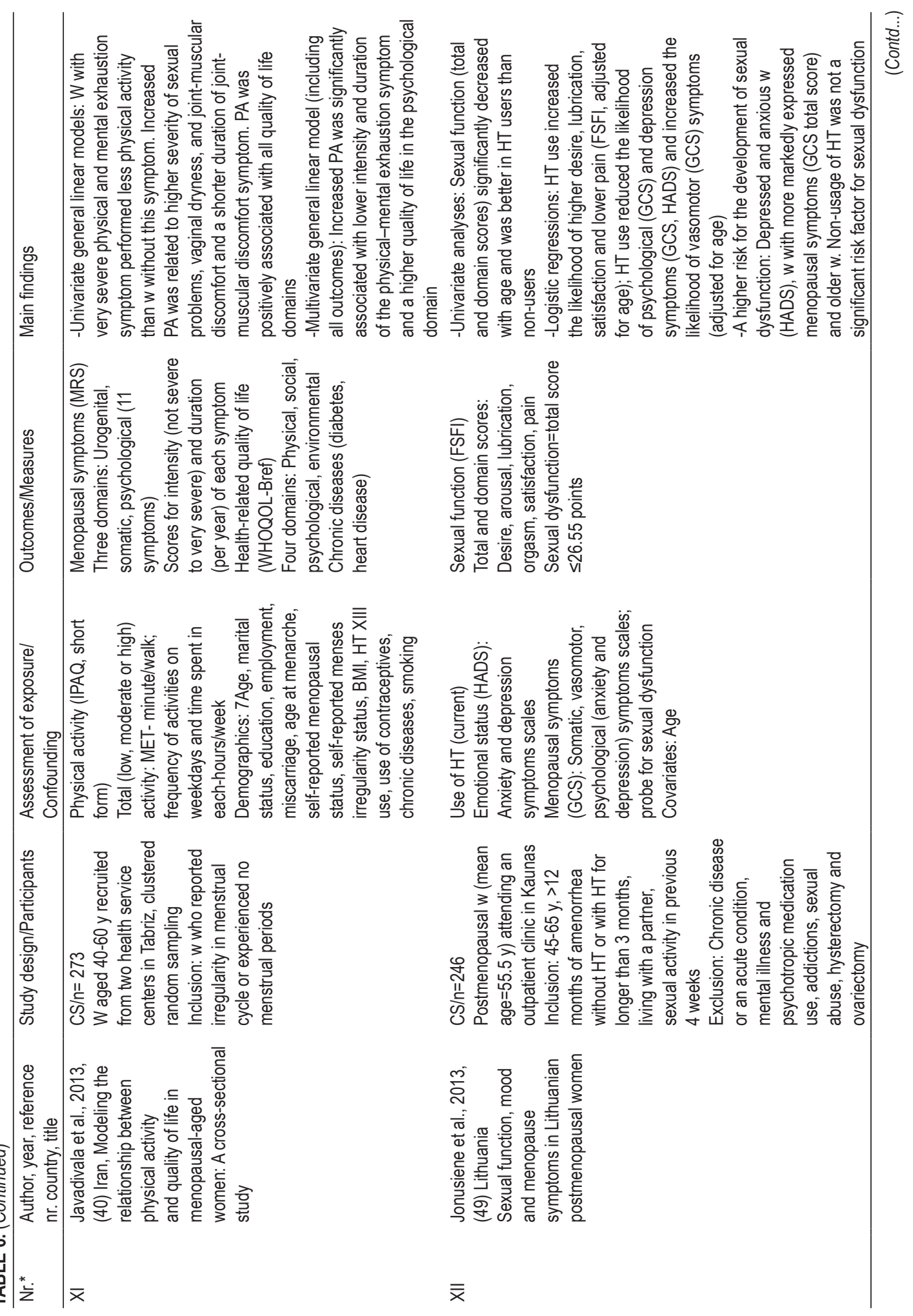




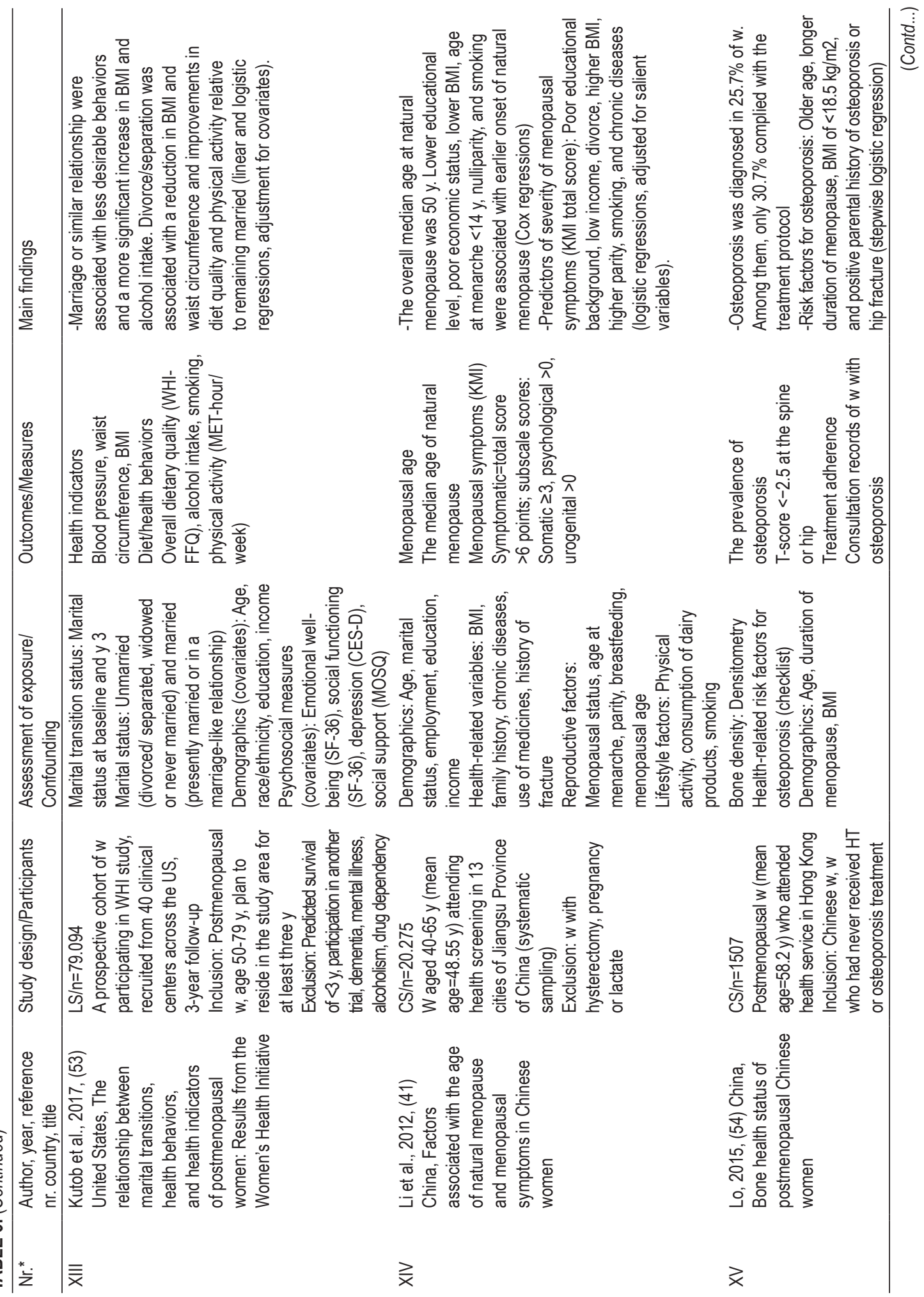




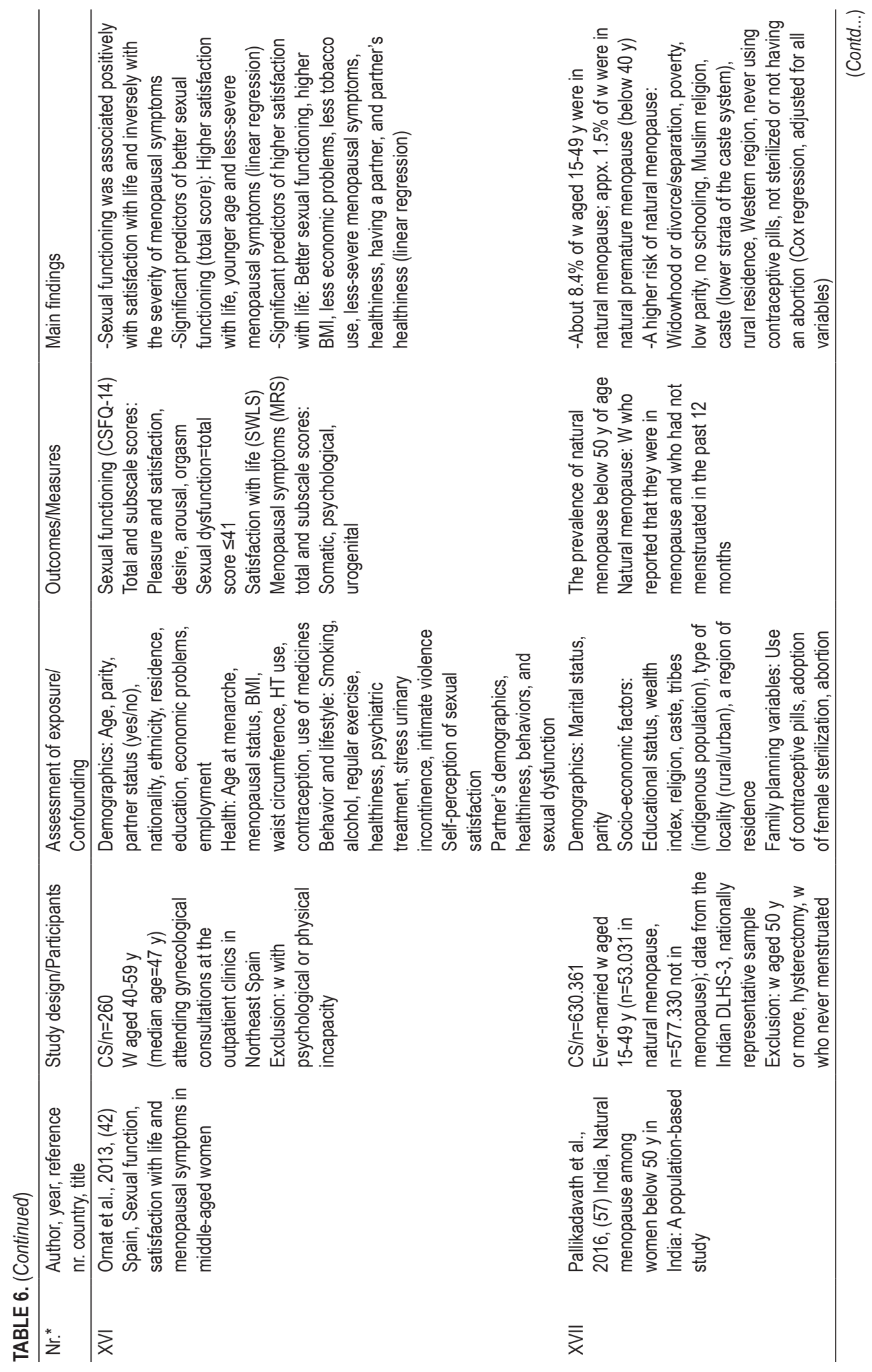




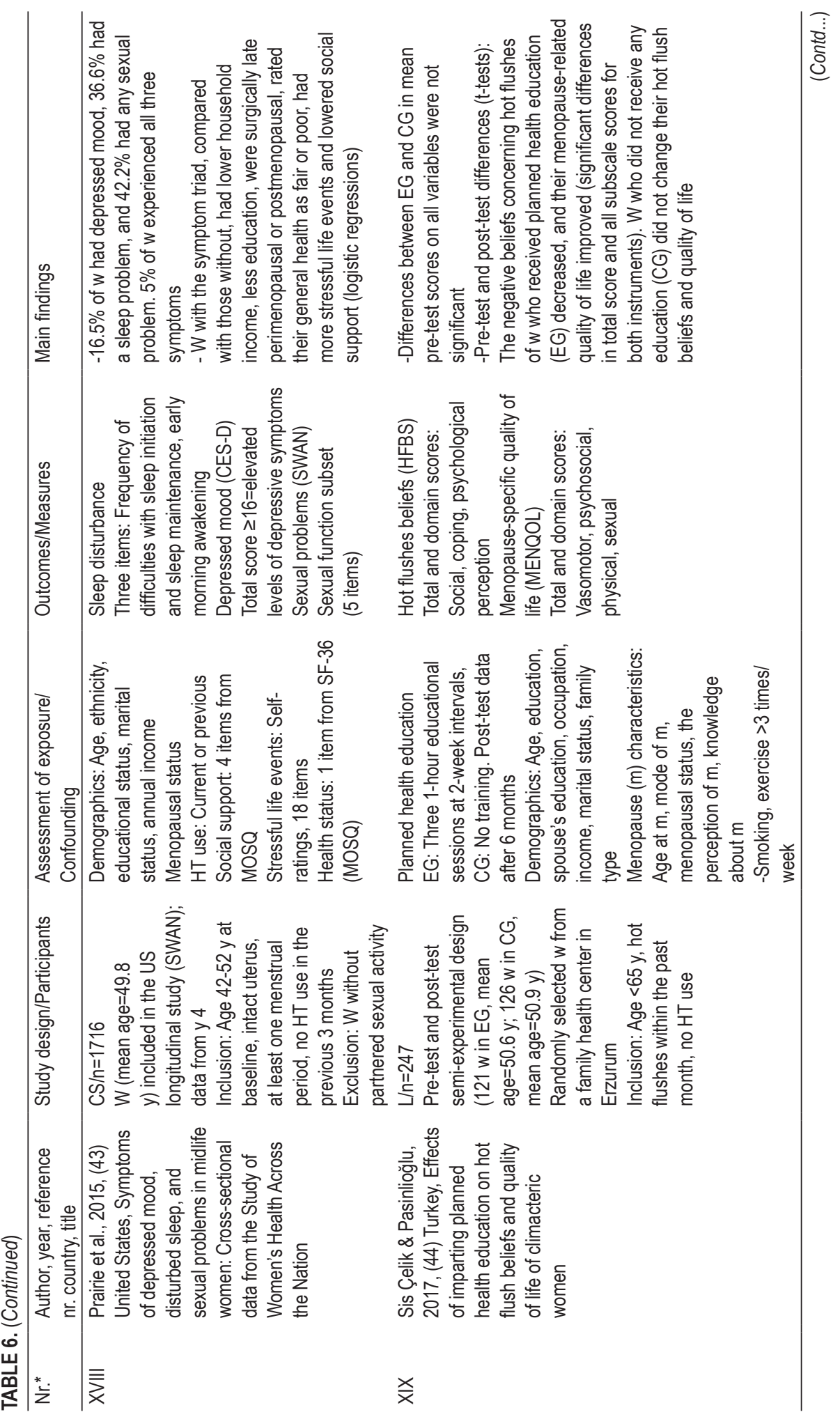




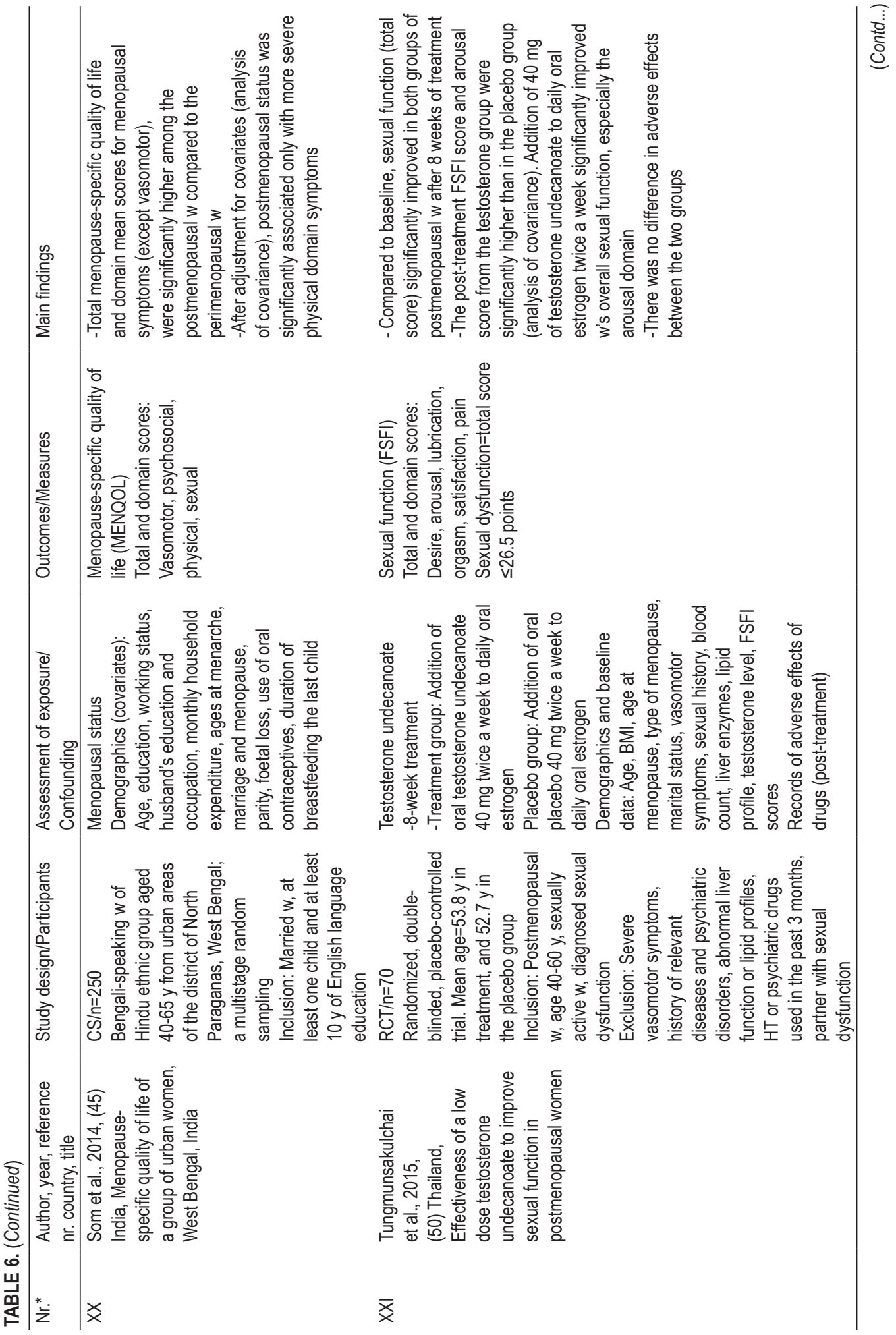




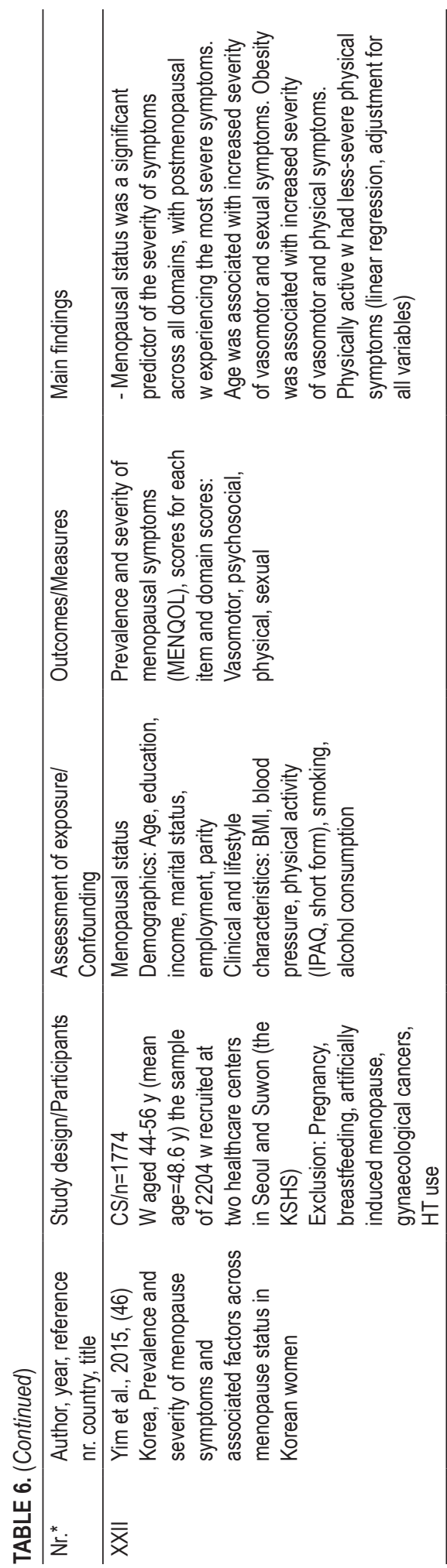

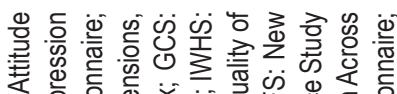

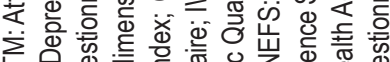

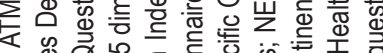
is 施.

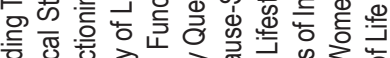

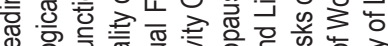

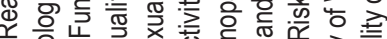

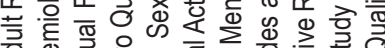

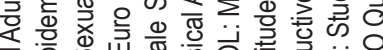
흔

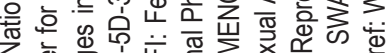

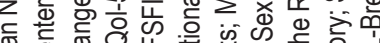

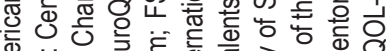

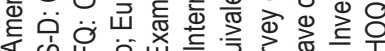

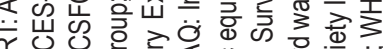

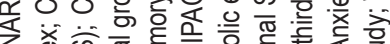

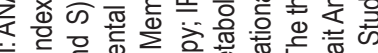

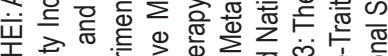

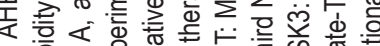
宁

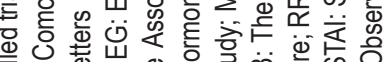

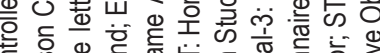

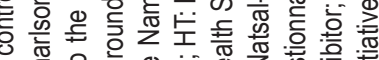

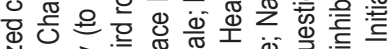

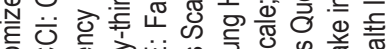

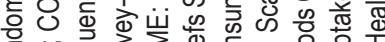

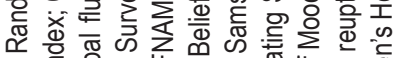

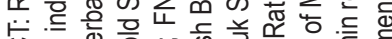

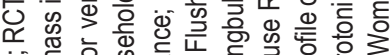

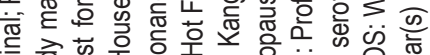

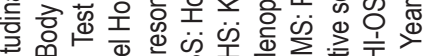

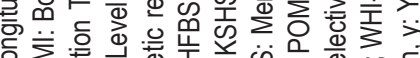

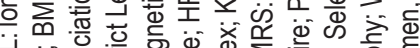

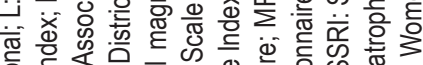

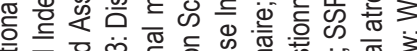
응 $\overline{\bar{J}}$ 흥

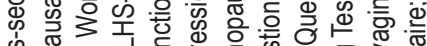

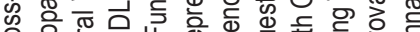

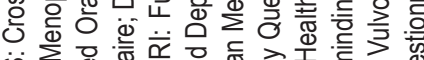

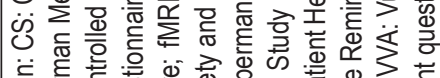

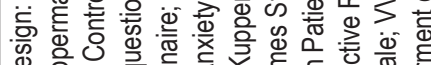

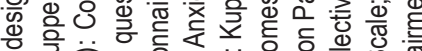

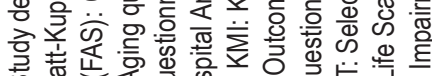

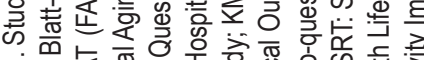

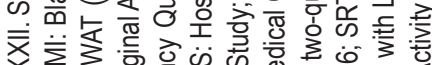

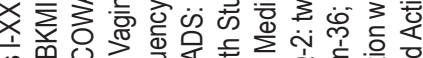
至

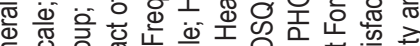

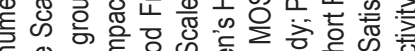

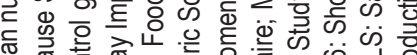

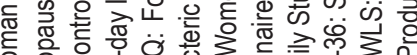

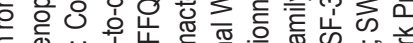

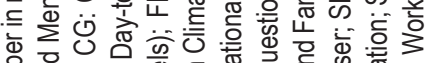

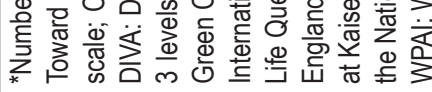


TABLE 7. Independent variables and/or covariates in 22 eligible studies

\begin{tabular}{|c|c|}
\hline Independent variables/covariates & $\begin{array}{c}\text { Study number in roman numerals } \\
\text { I-XXII }\end{array}$ \\
\hline \multicolumn{2}{|l|}{ Sociodemographic variables } \\
\hline Age (years or age groups) & I-XXII \\
\hline Age at marriage & $X X$ \\
\hline Cohort & $x$ \\
\hline Country/region & $\mathrm{XVII}$ \\
\hline Education & I-IV, VI-XI, XIII-XIV, VI-XX, XXII \\
\hline Family type & XIX \\
\hline Income & II-IV, VII, XIII-XIV, XVI-XX, XXII \\
\hline Marital/relationship status & I-II, IV-VIII, X, XI, XIII-XIV, XVII-XIX, XXII \\
\hline Nationality/country origin & XVI \\
\hline Occupation/employment & I, XI, XIV, XVI, XIX, XX, XXII \\
\hline Parental socioeconomic status & IX \\
\hline Partner's age & $\mathrm{XVI}$ \\
\hline Partner's/husband's education & VII, XVI, XIX, XX \\
\hline Partner's/husband's occupation & XVI, XX \\
\hline Partner's nationality and ethnicity & $\mathrm{XVI}$ \\
\hline Place of residency & I, VI, XVI-XVII \\
\hline Race/ethnicity & II, VIII-X, XIII, XVI, XVIII \\
\hline Religious affiliation & $\mathrm{XVII}$ \\
\hline Caste (low strata of the caste system) & $\mathrm{XVII}$ \\
\hline Sex & IX (results for women abstracted) \\
\hline \multicolumn{2}{|l|}{ Reproductive histories } \\
\hline Age at first birth & III \\
\hline Age at menarche & I, III, XIV, XVI, XX \\
\hline Age at menopause & I, XIV, XV, XIX-XXI \\
\hline Duration of menopause & $\mathrm{x}$ \\
\hline Bilateral oophorectomy & VI, VIII \\
\hline Breastfeeding/duration of breastfeeding & $X I V, X X$ \\
\hline Ever been sterilized & XVII \\
\hline Hysterectomy & II, VI \\
\hline Induced abortions & $\mathrm{XVII}$ \\
\hline Menopausal status & I-VI, IX, XIV, XVI, XVIII-XX, XXII \\
\hline Miscarriage/foetal loss & $\mathrm{XI}, \mathrm{XX}$ \\
\hline Parity & I, III, VII, XIV, XVI-XVII, XX, XXII \\
\hline Type of menopause & XVIII, XIX, XXI \\
\hline Use of contraceptives & XI, XVII, XX \\
\hline \multicolumn{2}{|l|}{ Hormonal therapy } \\
\hline Addition of testosterone to daily oral estrogen & $X X I$ \\
\hline Duration of hormonal therapy & XII \\
\hline Use of estrogen (vaginal and systemic) and SSSRIs & VIII \\
\hline Use of hormonal therapy (past or current) & XI-XII, XV-XVI, XVIII \\
\hline \multicolumn{2}{|l|}{ Sex hormones } \\
\hline Sex steroids, sex hormone-binding globulin, free androgen index & $X$ \\
\hline
\end{tabular}


TABLE 7. (Continued)

Independent variables/covariates

Study number in roman numerals

I-XXII

Sexual function

The assessment of whether health condition or disability affected sex life

Partner's sexual dysfunction

$\mathrm{V}$

Seeking help or advice about a sex life

Sexual activity

Sexual history

VIII

Sexual satisfaction

$X X I$

Clinical characteristics

$\mathrm{XVI}$

Abdominal circumference

$X V I$

Body mass index

I-VI, VIII, IX, XI, XIV-XVI, XXI-XXII

Bone density

$\mathrm{XV}$

Family history

$X I V-X V$

Functional impairment, work and activity impairment (WPAl)

IV-V

General health status

Health-related risk factors for osteoporosis

$\mathrm{V}, \mathrm{VIII}, \mathrm{XVI}, \mathrm{XVIII}$

$X V$

History of fracture

$X I V-X V$

Hypertension status

$I I I-X X$

Intimate violence

$\mathrm{XVI}$

Longstanding illness or disability

$\mathrm{V}$

Number or presence of various diseases

Partner's healthiness

IV-V, VII-VIII, X, XIV

$\mathrm{XVI}$

Psychiatric treatment

$X V I$

Stress urinary incontinence

$X \mathrm{VI}$

Use of medicines

Waist-to-hip ratio

$\mathrm{XIV}, \mathrm{XVI}$

$\mathrm{X}$

Menopausal symptoms

Climacteric symptoms (BKMI)

॥

XII

IV

Vulvovaginal atrophy and vasomotor symptoms 'vaginal dryness'and 'hot flushes' items on the MRS)

Knowledge and education about menopause

Perception of menopause

XIX

Knowledge about menopause

$\mathrm{XIX}$

Planned health education

$\mathrm{XIX}$

Behavioral/lifestyle characteristics

Alcohol consumption

Consumption of: Vegetables, fruits, red tea, Arabic coffee

Consumption of dairy products

$\mathrm{V}, \mathrm{VI}, \mathrm{XVI}, \mathrm{XXI}$

Partner's behavioral and lifestyle characteristics

XIV

$\mathrm{XVI}$

Physical activity (IPAQ, short form)

$\|, X I, X X\|$

Physical activity/exercise

Sitting and walking time

IV, XIV-XVI, XIX

III

Smoking

II, IV, VI, XI, XIV-XVI, XIX, XXII

Neuropsychological assessments

IX 
TABLE 7. (Continued)

\begin{tabular}{lc}
\hline Independent variables/covariates & $\begin{array}{c}\text { Study number in roman } \\
\text { I-XXII }\end{array}$ \\
\hline Psychosocial characteristics & IX, XII \\
Anxiety (STAI, HADS) & $\mathrm{V}, \mathrm{XII-XIII}$ \\
Depression symptoms (PHQ-2, HADS, CES-D) & $\mathrm{XIII}$ \\
Emotional well-being and social functioning (SF-36) & $\mathrm{XIX}$ \\
Mood profile (POMS) & II \\
Quality of life (WHOQOL-Bref) & $\mathrm{XII-XIII}$ \\
Social support (MOSQ) & $\mathrm{XVIII}$ \\
Stressful life events
\end{tabular}

BKMI: Blatt-Kupperman Menopausal Index; CCl: Charlson Comorbidity Index; CES-D: Center for Epidemiological Studies Depression; GCS: Greene Climacteric Scale; HADS: Hospital Anxiety and Depression Scale; IPAQ: International Physical Activity Questionnaires; IQ: intelligence quotient; MOSQ: Medical Outcomes Study Questionnaire; MRS: Menopause Rating Scale; PHQ-2: two-question Patient Health Questionnaire; POMS: Profile of Moods States Questionnaire; SF-36: Short Form-36; SSRI: selective serotonin reuptake inhibitor; STAI: State-Trait Anxiety Inventory; WHOQOL-Bref: Word Health Organisation Quality of Life-26 questionnaire; WPAIQ: Work Productivity and Activity Impairment questionnaire

Australia (40); Brazil (26,58); China (42,55); India $(46,60)$; Iran (41,59); Korea (47); Lithuania (50); the Netherlands (53); Saudi Arabia (39); Spain (43); Thailand (51); Turkey (45); the United Kingdom (49); and the United States $(44,52,54,57)$. One study $(56)$ included women from the United States and five Western European countries (France, Germany, Italy, Spain, and the United Kingdom).

\section{Description of study participants}

The age of the study participants varied depending on the different aims and inclusion criteria of the included studies, ranging between 15 and 81 years of age. Most studies included women aged $40-65$ years or similar $(40-43,46,48,50,51,58)$. Two studies included participants from their mid-40s to their mid-50s $(47,57)$, while three studies included middle-aged women and older whose differences in age ranged from 30 to 40 years $(52,54,56)$. One study that examined the age of natural menopause (60) included women aged 15-49 years, while three studies $(44,55,59)$ did not state the age range of the participants, and three studies reported only lower or upper age limit $(39,45,53)$. Participants in one study were men and women aged 16-74 years, and we abstracted data available for women aged 45-64 years who reported on their menopausal status (49). Only nine studies reported the mean age of all the participants $(39,40,42,44,47,50,53,55,59)$ and three studies $(45,46,51)$ reported the mean age of women in specific subgroups, but not in the total sample.

Eight studies included only postmenopausal women (50-56,59), three studies enrolled premenopausal and menopausal women $(48,49,60)$, and two studies included peri- and postmenopausal women $(41,46)$. Nine studies enrolled pre-, peri-, and postmenopausal women $(39,40,42-45,47,57,58)$. In all studies, amenorrhea for longer than 12 months was the criterion for menopause, while irregular menstruation within the previous 12 months was the criterion for perimenopause. Menopausal status was diagnosed based on women's reproductive histories in all studies, and one study also included hormonal evaluations (57). Study participants included women who had either natural or surgically-induced menopause. Based on the inclusion/exclusion criteria related to the type of menopause, four studies included women in natural menopause $(47,53,59,60)$ and two studies excluded women who had undergone bilateral oophorectomy $(39,58)$. Three studies examined the effects of type of menopause and enrolled naturally and surgically menopausal women $(44,45,51)$. The effects of bilateral oophorectomy were controlled in the data analyses of two studies $(40,52)$. Five studies did not specify the type of ovariectomy as an exclusion criterion (50), exclude women based on hysterectomy $(42,44,50)$, or control for the effects of hysterectomy $(40,48)$. Eight studies did not include variables related to the mode 
of menopause (41,43,46,49,54-57). Seven studies stated that women using hormonal therapy (HT) had been excluded $(39,45,47,48,51,55,59)$, one study included women who had not been on HT in the previous 3 months (44), and one study included women who were not on HT and those who had been on HT for longer than 3 months (50).

\section{Assessment of menopausal symptoms}

Nine studies assessed menopausal symptoms as either one of the outcomes or the main outcome (39-47), and five studies assessed menopausal symptoms as predictors of other outcomes $(48,50,52,56,59)$. Eleven studies used a validated tool for assessing the frequency and/or severity of menopause-related symptoms: Specifically, the Menopause Rating Scale (MRS) (39,41,43); the Menopause-Specific Quality of Life Questionnaire (MENQOL) (40,45-47,59); the Blatt-Kupperman Menopausal Index (KMI) $(42,56)$; or the Greene Climacteric Scale (GCS) (50). Of these, three studies provided results for total and/or each symptom score(s) $(41,48,59)$, while menopausal symptoms presented in other studies were classified based on the validated scales as somatic, urogenital, and psychological (MRS and KMI); somatic, vasomotor, and psychological (GCS); or vasomotor, physical, sexual, and psychological (MENQOL). One study (44) examined the symptom triad during the menopausal transition; this included the assessment of sleep disturbance and sexual function using subsets of questions from the questionnaire applied in the study of Women's Health Across the Nation, as well as the assessment of depressed mood with a validated instrument (the Center for Epidemiological Studies Depression [CES-D]). Two studies assessed vulvovaginal atrophy symptoms (56) and hot flushes $(52,56)$, with one item only.

\section{RESULTS}

\section{Socio-demographic predictors of menopausal symptoms}

The eligible studies included a wide range of potential determinants of menopausal symptoms. The most frequent independent variables in studies with menopausal symptoms as outcomes were educational status (39-60) and women's age (39-60).
Other frequently assessed variables were those related to economic status: Women's occupation or employment (39,41-43,45-47); family income $(42,44,45,47)$ or expenditure $(46)$; economic problems (43); and residence status (39). The effects of marital or relationship status were examined in six studies $(39-42,44,45)$. Some studies also controlled for partner's demographic, lifestyle, and clinical characteristics (43) or husband's educational level $(45,46)$, and occupation (46). Significant effects of age, independent of menopausal status and other relevant variables, were found in two studies. Women from Saudi Arabia aged 50-54 years had a higher risk of experiencing moderate/severe urogenital symptoms (measured with the MRS) (39), while a study of Korean women found a significant positive association between women's age and severity of vasomotor and sexual symptoms, measured with the MENQOL (47). Three studies demonstrated that women's educational level was inversely related to the severity of menopausal symptoms, independent of other variables. Studies conducted in the United States $(40,44)$ showed that lower educational status was associated with a higher risk of experiencing moderate to severe symptoms in the vasomotor domain of the MENQOL (40) and the symptom triad consisting of sleep disturbances, depressed mood, and sexual problems (44). One study in China found that poor educational background was associated with higher total symptom scores, as well as with the physical and urogenital domain scores of the KMI. This study also showed that total scores and each subscale score of the KMI significantly increased for separation or divorce (42). Some studies also found significant independent effects of different indicators of economic status on menopausal symptoms. Women who generally experienced more severe menopausal symptoms as well as urogenital symptoms (42), and women with the symptom triad (44), lived in households with lower income. A higher incidence of moderate to severe urogenital symptoms was also found for homemakers and women living in apartments or small houses compared to working women and women living in large houses (39). Menopausal symptoms were assessed with the MENQOL as predictors of women's attitudes toward menopause in one study with postmenopausal women in Iran (59). This study 
also focused on the relationships between the severity of menopausal symptoms in different domains (the MENQOL) and various demographic and health characteristics. Univariate analyses showed that the educational level of the husband, but not the woman herself, was related to menopausal symptoms. Women who had an illiterate husband reported significantly more vasomotor and physical symptoms than women whose husband had at least some primary education.

\section{Reproductive factors as determinants of menopausal symptoms}

Seven studies examined the effects of menopausal status on the severity of menopausal symptoms and compared subgroups of pre-, peri-, and postmenopausal women $(39,40,42-44,46,47)$, while in two studies menopausal status was included either as an inclusion variable (41) or for comparison of baseline characteristics between the experimental and control groups (45). Three studies additionally classified postmenopausal women into subgroups according to the duration of this reproductive stage (45), women's age (40) or the type of menopause (44), two studies differentiated between subgroups of early and late perimenopausal women $(44,47)$, and two studies included only peri- and postmenopausal women $(41,46)$. Six studies found significant independent effects of menopausal status on the severity of menopausal symptoms $(39,40,43,44,46,47)$, but their results differed for specific symptom domains. Postmenopausal status was associated with a higher risk of experiencing more severe menopausal symptoms in all studies except one (39). Gartoulla et al. (40) examined predictors of the vasomotor domain symptoms assessed with the MENQOL; the highest likelihood for any and moderate to severe vasomotor symptoms was found in postmenopausal women under 55 years of age. Another study in India (46), using the same instrument, found that vasomotor domain scores were not related to menopausal status, while the total menopause-specific quality of life mean scores - and those in the physical, sexual, and psychological domains - were significantly higher among the postmenopausal women than the perimenopausal women. However, after adjustment for covariates, postmenopausal status was significantly associated only with more severe physical domain symptoms. Among women in Korea, menopause status showed significant independent effects on the severity of symptoms across all domains of the MENQOL, with postmenopausal women experiencing the most severe symptoms. The most significant increase of severity of menopausal symptoms and the most significant differences between menopausal status groups were observed in vasomotor and sexual domain scores (47). Postmenopausal status was associated with more severe total, somatic and urogenital symptoms assessed by the MRS among women in Spain (43). Praire et al. (44) found a significant independent effect of menopause status on the occurrence of the symptom triad among women in the United States. Late perimenopausal and surgically postmenopausal women experienced symptom triad sleep disturbance, depressed mood and sexual problems more often than premenopausal, early perimenopausal and naturally menopausal women. The results of a study of women living in Saudi Arabia showed that perimenopausal women had a higher risk of experiencing moderate to severe somatic symptoms compared to pre- and postmenopausal women (39), while the effects of menopausal status on total symptoms experienced as well as on urogenital and psychological symptoms were not significant. A study of Chinese women found that menopausal status was not significantly associated with menopausal symptoms assessed by the KMI (42). One intervention study in Turkey showed that women who received planned health education related to the climacteric period improved their menopause-related quality of life (significant differences in their MENQOL total score and all subscale scores). These effects were independent of women's perception of menopause, the previous knowledge about menopause and other relevant factors (45). Apart from menopausal status, parity was the most frequently examined independent variable $(39,42,43,46,47)$. Two studies demonstrated that higher parity was associated with more severe menopausal symptoms, but the effects varied according to the symptom domains and instruments used. Among women from Saudi Arabia, nulliparous women had nearly 3 times more risk of experiencing severe/moderate somatic symptoms measured with the MRS compared to women who became pregnant 5 times or more (39). Another study in China showed that women 
obtained significantly higher total scores on the KMI, as well as scores on somatic and urogenital subscales when the parity was 2 times higher or more (42). Four studies examined the effects of age at menarche $(39,42,43,46)$ and age at menopause $(39,42,45,46)$ and found no significant relationships between these variables and the severity of menopausal symptoms. Studies that examined various menopausal symptoms as outcomes rarely controlled for HT use and type of menopause. Women on HT were excluded in three studies $(39,45,47)$. Some studies also excluded women with a bilateral oophorectomy under 50 years of age (39), women with a hysterectomy (42), and women with artificial menopause due to hysterectomy or radiation (47). One study included a hysterectomy and bilateral oophorectomy as independent variables. Hysterectomy was associated with a higher likelihood of moderate to severe vasomotor symptoms, but this effect was not significant when the relationships with other relevant variables were controlled for in a logistic regression analysis (40). Pregnancy or breastfeeding was an exclusion variable in one study (47), and two studies that examined the effects of breastfeeding $(42,46)$ found no significant relation between this variable and menopausal symptoms.

\section{Clinical characteristics of the participants associated with menopausal symptoms}

Five studies included BMI as an independent variable $(39,40,42,43,47)$ and found significant effects on menopausal symptoms, while one study provided only descriptive data on BMI (11). AlQuaiz et al. (39) found that obese women were at higher risk of experiencing more severe psychological symptoms compared to normal and overweight women. Increased BMI, measured as a continuous variable, was a significant predictor of the total MRS score. Another study also found significant positive correlations between BMI and MRS total, somatic, and urogenital scores. However, this study did not examine predictors of menopausal symptoms in a multivariate model, controlling for other significant relationships (43). Higher BMI was associated with higher total and all three subscale scorings of the KMI (after adjustment for confounding factors) (42). Two studies showed that BMI is also a significant predictor of menopausal symptoms measured with the MENQOL. A BMI that is usually classified as "overweight" $\left(25-29.9 \mathrm{~kg} / \mathrm{m}^{2}\right)$ was associated with a higher risk of experiencing moderate to severe vasomotor symptoms, while obese women (a BMI of $\geq 40 \mathrm{~kg} / \mathrm{m}^{2}$ ) were at higher risk of experiencing any vasomotor symptoms (40). Another study of women in Korea showed that obesity was positively associated with severity of vasomotor and physical symptoms. This study classified BMI according to Asian-specific criteria, with a BMI of $25 \mathrm{~kg} / \mathrm{m}^{2}$ or higher considered "obese" (47). Two studies found significant effects of other clinical characteristics on menopausal symptoms. History of chronic diseases was a significant predictor of higher total and all three subscales scores of the KMI (42). The symptom triad of sleep disturbance, depressed mood, and sexual problems occurred most often among women who had experienced more stressful life events, had lower social support and rated their general health as fragile (44). One study that assessed menopausal symptoms as predictors of sexual dysfunction found significant associations of current hormone therapy (HT) use and menopausal symptoms (of the GCS), adjusted for age. HT use reduced the likelihood of total psychological domain symptoms and depression subdomain symptoms but increased the likelihood of vasomotor symptoms (50).

\section{Behavioral and lifestyle characteristics as predictors of menopausal symptoms}

Six studies measured women's physical activity either with a standard validated instrument, i.e., the International Physical Activity QuestionnaireShort Form $(41,47)$ or with a scale that included the assessment of weekly physical activity or exercise $(39,42,43,45)$. Among them, four studies examined independent effects of this variable on menopausal symptoms, while two studies used physical exercise data only for comparison of sample subgroups (45) or for a description of respondents' characteristics (43). Three studies found significant independent effects of physical activity on different symptoms or domains, with women who regularly exercised experiencing less-severe menopausal symptoms. Among women in Saudi Arabia, those regularly performing physical activity had significantly less-severe somatic, urogenital and total MRS symptoms compared to women who did not 
exercise or exercised irregularly (39). Another study, in Iran, examined the effects of physical activity on each symptom of the MRS. Multivariate analysis showed that women who had more physical activity had less-severe physical and mental exhaustion symptom (41). Moderate or high physical activity was also associated with less-severe physical symptoms on the MENQOL among Korean women (47). Other behavioral and lifestyle characteristics examined were smoking (40-43,45,47), alcohol consumption $(40,43,47)$, and dietary habits $(39,42)$. One study that included measures of various characteristics of women and their partners provided only descriptive data for tobacco and alcohol use (43), and one study compared baseline differences in smoking habits among women in the experimental and control group (45). Studies did not find significant effects of alcohol consumption on menopausal symptoms $(40,47)$. Smoking was associated with greater likelihood of experiencing any vasomotor symptoms and moderate to severe vasomotor symptoms of the MENQOL (40), as well as with more severe total, and domain-specific, symptoms measured with the KMI (42). A study with Saudi Arabian women found that intake of Arabic coffee significantly predicted the severity of overall menopausal symptoms measured with the MRS and symptoms in the urogenital domain (39).

\section{The prevalence of menopausal symptoms}

Five studies provided data on the prevalence of menopause-related symptoms in different countries, and all studies except one (43) included large samples of women. These studies showed that the incidence of menopausal symptoms, assessed with different scales, is highest among postmenopausal women - especially the incidence of sexual problems. The most frequent symptoms ( $>50 \%)$ assessed with the KMI in postmenopausal women in China were sexual problems, muscle/joint pain, and insomnia. Sexual problems were reported significantly more frequently in postmenopausal than in pre- and perimenopausal women (42). A study with Australian women showed that the prevalence of any vasomotor symptoms assessed with the MENQOL ranged from $32.7 \%$ in premenopausal women to $74.2 \%$ in postmenopausal women younger than 55 years. The prevalence of sexual symptoms ranged from $43.5 \%$ in premenopausal women to $68 \%$ in postmenopausal women. Vasomotor symptoms persisted in $42.1 \%$ and sexual symptoms persisted in $62.5 \%$ of women aged between 60 and 65 years. This study also showed that nearly $10 \%$ of women aged $60-65$ years were current HT users, while the use of non-hormonal prescription therapies with proven efficacy was low (40). Among women in Korea, the highest prevalence of most of the menopause symptoms in the MENQOL (18 of 29 items) was found among postmenopausal women. The mean domain scores showed that the most bothersome symptoms for perimenopausal women were in the physical domain, while postmenopausal women were most bothered by sexual symptoms (47). The study that identified a symptom triad related to the menopausal transition among women in the United States also provided data on the prevalence of these symptoms: About 16.5\% of women had CES-D scores indicating a high depressive symptom burden, $36.6 \%$ had a sleep problem, and $42.2 \%$ had a sexual problem; only $5 \%$ of women experienced all three symptoms (44). Finally, a study in Spain with a smaller sample of 260 women aged 40-59 years found that $14.6 \%$ of women in the total sample had severe menopausal symptoms assessed with the MRS. The incidence of severe menopausal symptoms was the highest (39.5\%) in postmenopausal women who did not use hormonal contraception (44).

\section{Sexual functioning}

Some of the abovementioned studies examined the determinants of menopausal symptoms in women. The urogenital scales of the MRS and the KMI include sexual problem items and vaginal dryness items that are classified as sexual symptoms in some studies. The results of four studies that assessed sexual symptoms with a sexual subscale of the MENQOL are described in the previous section (40,45-47). Seven independent studies examined various determinants of sexual functioning and sexual lifestyles $(43,44,48-52)$. Four studies assessed sexual functioning and diagnosed sexual dysfunction using standard questionnaires, such as the Female Sexual Function Index (FSFI) $(48,50,51)$ and the 14-item Changes in Sexual Functioning Questionnaire 
(CSFQ) (43). Three studies analyzed subsets of data from questionnaires applied in national studies. One of these included postmenopausal women with at least one vaginal symptom (e.g., dryness, itching, irritation, soreness, or pain during sexual activity). Respondents reported the impact of vaginal symptoms (in the past 4 weeks) using the sexual functioning subscale of the Day-to-Day Impact of Vaginal Aging (DIVA) questionnaire. The questionnaire had slightly different versions for sexually active and inactive women (52). Another study examined sexual function problems as part of the menopause-related symptom triad, using the sexual function subset of the questionnaire applied in the Study of Women's Health Across the Nation. This subset included five questions on desire, arousal, satisfaction, orgasm, and vaginal dryness (44). Significant predictors of the symptom triad are described in the previous section. Finally, Field et al. (49) analyzed data on sexual lifestyles from the third National Survey of Sexual Attitudes and Lifestyles in Britain. These included questions on sexual activity in the past 4 weeks, sexual satisfaction in the past year, and sexual problems due to vaginal dryness.

\section{Socio-demographic predictors of sexual functioning}

Two of the studies that applied the FSFI examined the effects of various socio-demographic characteristics of the respondents on their sexual function and sexual dysfunction $(48,50)$, while the third study (with a randomized controlled trial design) included relevant demographic variables for the comparison of treatment and placebo groups (51). A study in Brazil that included healthy women aged 40-65 found significant associations with sexual dysfunction for women aged 56-65, women with lower education, and divorced or separated women. However, in a logistic regression with other relevant predictors, significant independent effects were found only for age, with women aged 56-65 at higher risk of sexual dysfunction (48). This study diagnosed sexual dysfunction with the FSFI (total score of $\leq 26.55$ points). Another study in Lithuania also found a significant independent effect of age, with older women being at higher risk for the development of sexual dysfunction, diagnosed with the same instrument (50).
Similarly, among women in Spain (43), older age was a significant independent predictor of worse sexual functioning (lower total CSFQ-14 score). A study in the United States (52) examined the effects of selected demographic characteristics on each of the DIVA domain scales (adjusted for HT use, other demographic variables, and clinical variables). Results for sexually active women (partnered or unpartnered sexual activity) showed that vaginal symptoms had lower sexual function impact scores among Latina/Hispanic women compared to NonLatina women. Women who had been sexually inactive in the past 3 months, older women and women with a spouse or sexual partner reported higher scores on the sexual function impact scale.

\section{Reproductive variables associated with sexual functioning}

Three studies included only postmenopausal women (50-52), and three studies examined the effects of reproductive stages on sexual functioning $(43,48)$ and sexual lifestyles $(49)$. The significant independent effect of menopausal status was found in a study on sexual lifestyles in Britain, which showed that sexual activity was less frequent and vaginal dryness (a sexual response problem) was more common in postmenopausal women aged 54-65 than in premenopausal women (after adjustment for age, relationship status, and comorbidities). A study in Brazil found that menopausal status and hysterectomy were significantly associated with sexual dysfunction among healthy women, but these associations were not significant after adjustment for confounding factors in a logistic regression analysis (48). Similarly, a study with women in Spain showed that postmenopausal status was associated with worse sexual functioning and more severe menopausal symptoms. However, menopausal status was not a significant predictor of sexual functioning when relationships with other relevant variables were controlled for in a linear regression analysis (43).

\section{Clinical variables associated with sexual functioning}

Three studies demonstrated significant independent effects of menopausal symptoms on women's sexual functioning, assessed with different instruments. 
These studies included healthy women and/or excluded women with comorbidities. Moderate to severe climacteric symptoms measured with the Blatt-Kupperman Menopausal Index showed significant independent effects on sexual dysfunction (diagnosed with the FSFI) among women in Brazil (48). A study with postmenopausal women in Lithuania that used the same instrument for the assessment of sexual dysfunction (50) also found a higher risk for the development of sexual dysfunction among women with more markedly expressed menopausal symptoms (total GCS score). A study with 260 women in Spain who attended gynecological consultations at outpatient clinics demonstrated that less-severe menopausal symptoms measured with the MRS had a significant independent effect on better sexual functioning; this study found that the prevalence of sexual dysfunction diagnosed with the CSFQ-14 was $46.5 \%$ in this sample (43). One study in the United States (52) showed that, among women who were sexually active (engaging in either partnered or unpartnered sexual activity), the impact of vaginal symptoms (measured by the sexual function domain of the DIVA) was higher for women who reported at least moderately bothersome hot flushes in the previous months (after adjustment for HT use, demographic, and clinical variables).

Two studies found that depression measured with the HUDS subscale was a significant independent predictor of worse sexual functioning. Women with comorbid depression were at higher risk of developing sexual dysfunction (diagnosed with the FSFI) (50) and reported a more significant impact of vaginal symptoms (measured with the DIVA sexual function subscale) compared to women without comorbid depression (52). Higher risk for the development of sexual dysfunction was also associated with more markedly expressed anxiety (measured with the HUDS subscale) among postmenopausal women (50). One study found a significant effect (adjusted for age) of the current use of HT on the sexual functioning of postmenopausal women. HT use increased the likelihood of higher desire, lubrication, satisfaction, and lower pain measured with the FSFI. Non-use of HT was not a significant risk factor for sexual dysfunction (50). A study in Thailand (51) with a randomized controlled trial design demonstrated the effectiveness of testosterone therapy for improving sexual functioning in postmenopausal women with sexual dysfunction. Treatment for 8 weeks with $40 \mathrm{mg}$ of testosterone undecanoate and daily oral estrogen twice a week was associated with a significant improvement in women's overall sexual functioning and arousal domain (on the FSFI) than the use of estrogen alone; there were no differences in adverse effects between the treatment and placebo groups. General health status has significant effects on sexual activity and satisfaction. A study with a representative sample of men and women aged 16-74 years in Britain (49) showed that poor health was generally associated with decreased sexual activity and satisfaction among men and women at all ages. Women aged 45-64 years who reported bad or terrible health engaged significantly less often in sexual activity than women with excellent and perfect health (after adjustment for relationship status). Only $20.6 \%$ of postmenopausal women reporting a health condition or disability affecting their sex life in the past year had sought clinical advice. A study in the United States (52) showed that sexual activity was a significant independent predictor of the impact of vaginal symptoms (assessed with the DIVA's sexual function domain), with lower impact reported among women who were sexually active on at least a weekly basis compared to non-sexually active women and those who were sexually active less than weekly.

\section{Behavioral and lifestyle characteristics associated with sexual functioning}

Studies also found that physical activity, a better quality of life and satisfaction with life are positively associated with sexual functioning. Sedentary women and women with a low and average quality of life (measured with the WHO Quality of Life 26 questionnaire [WHOQOL-Bref]) were more likely to develop sexual dysfunction (diagnosed with the FSFI) compared to moderately and very active women and women with high quality of life, respectively (48). Similarly, higher satisfaction with life (measured with the SWLS) was a significant independent predictor of better sexual functioning, i.e. higher total CSFQ-14 scores (43). 


\section{Health indicators and health behaviors}

Two studies examined health indicators as outcomes (i.e., cardiovascular health (53) and osteoporosis (55) in postmenopausal women), one study (54) examined the effects of marital transitions after menopause on various health indicators and health behaviors; and one study examined physical activity in women with diabetes and heart disease (41). Jaspers et al. assessed the association between oestradiol, testosterone, sex hormone-binding globulin (SHBG), and free androgen index (FAI) with optimal cardiovascular health $(\mathrm{OCH})$ in men and naturally postmenopausal women who were on average 69.6 years of age (53). The findings related to naturally postmenopausal women indicate that sex steroids and SHBG might have a significant role in determining their cardiovascular health. Multivariate analysis showed that $\mathrm{OCH}$ in women was associated with lower levels of testosterone and FAI and higher levels of SHBG, irrespective of potential confounders (demographic and reproductive variables, chronic diseases, and other sex steroids). The definition of $\mathrm{OCH}$ was based on three health factors (total cholesterol, fasting glucose, and blood pressure) as well as on four health behaviors (physical activity, smoking, BMI, and diet). $\mathrm{OCH}$ was reported for $10.4 \%$ of women in this study. A study with peri- and postmenopausal women in Iran (41) found that women who had a history of cardiovascular disease and diabetes mellitus had significantly more physical activity compared to women without these chronic diseases, which may indicate that they were motivated to engage in physical activity to prevent the consequences of their diseases. The effect of physical activity was adjusted for women's quality of life and menopausal symptoms. This study also assessed relevant demographic and reproductive factors, BMI, HT use, and smoking, but provided only descriptive data for these variables. A study in Hong Kong with postmenopausal Chinese women (55) found that significant risk factors for osteoporosis were older age, longer duration of menopause, low BMI, and parental history of osteoporosis or hip fracture. Osteoporosis was diagnosed in $25.7 \%$ of women. Among these, less than one-third complied with treatment, while the other women discontinued or refused treatment. One longitudinal study in the United States examined the effects of marital transitions on health indicators (blood pressure, waist circumference, and BMI) and health behaviors (dietary quality, alcohol intake, smoking, and physical activity) among postmenopausal women (54).

Interestingly, the results showed that marital transitions after menopause are accompanied by modifiable health outcomes that were more favorable for women experiencing divorce or separation compared to women entering a new marriage. Divorce or separation after menopause was associated with a reduction in BMI and waist circumference and improvements in diet quality and physical activity, while transition into marriage or marriage-like relationships was associated with an increase in BMI and alcohol intake. In this study, the effects of marital status were adjusted for relevant covariates (other demographic variables, emotional well-being and social functioning, depression, and social support).

\section{Health-related quality of life}

Two studies examined the determinants of health-related quality of life assessed with standard questionnaires (e.g., the Euro Quality of Life [5 dimensions, 3 levels] (EuroQol-5D-3) (56) and WHOQOL-Bref (41)), one study examined the determinants of general satisfaction with a life measured with Diener's Satisfaction With Life Scale (43), and one study examined the impact of vaginal symptoms of menopause-related quality of life with the DIVA questionnaire (52). Factors associated with the DIVA sexual functioning domain were described earlier. In this section, findings related to other dimensions of the DIVA questionnaire are presented. The results of the study that assessed the impact of vaginal symptoms on health-related quality of life measured with the DIVA questionnaire showed significant effects of some clinical and demographic characteristics of the participants on the different quality of life dimensions (52). Multivariate analysis showed that women who had higher HADS depression subscale scores, or who were experiencing urinary incontinence on at least a weekly basis, reported higher impact of vaginal symptoms on all three domains (activities of daily living, emotional well-being, and self-concept/body image). Furthermore, the impact on activities included in the daily living domain was higher among women 
who reported only fair or poor overall health and among women who had higher BMI. Scores on the DIVA emotional well-being impact scale were lower among women with a spouse or sexual partner and higher among women who did not have a college degree. Scores reflecting the impact of vaginal symptoms on self-concept and body image domain items were lower among Latina/Hispanic women (compared with Non-Latina White women), older women, and women with diabetes. Another study analyzed data from the International Women's Health Study (56) and examined the association between vulvovaginal atrophy symptom severity and quality of life, after adjusting for demographics, health history, and health behaviors. The primary independent variable was assessed with only one item from the urogenital scale of the MRS (the vaginal dryness item). Quality of life was measured using the EuroQol-5D-3 instrument, which assesses the health state of the respondent at the time of completion based on five dimensions (mobility, selfcare, usual activities, pain/discomfort, and anxiety/ depression). The results showed that, after pooling data from all countries together, each incremental level of severity of vulvovaginal atrophy symptoms (none through severe) was associated with a significant decrement in the quality of life scores. One study showed that physical activity was positively associated with the environmental, social, and psychological health-related quality of life domains of the WHOQOL-Bref (41). Multivariate analysis showed that increased physical activity had independent effects only on the psychological quality of life domain when the relationships with other quality of life domains, menopausal symptoms, and chronic diseases were statistically controlled for; however, this study did not test the effects of potential socio-demographic confounders. A study in Spain with women aged 40-59 years showed that menopausal status was not associated with women's global satisfaction with life, while various demographic, lifestyle, and clinical characteristics were significantly associated with life satisfaction. A linear regression analysis showed that significant predictors of higher satisfaction with life were better sexual functioning; higher BMI; fewer economic problems; less tobacco use; less-severe menopausal symptoms; healthiness; having a partner; and partner's healthiness (43).

\section{Physical performance and cognitive changes}

Two studies found that menopausal transition affects women's physical performance and cognitive functioning $(57,58)$. Premenopausal women performed significantly better in grip strength and chair stands than peri- and postmenopausal women. However, the effect of menopausal status remained significant only for grip strength, when the effects of other relevant demographic and reproductive variables were controlled for in a linear regression analysis (58). Jacobs et al. (57) examined the effects of menopausal status on changes in memory circuitry during verbal encoding and memory retrieval tasks among women aged 45-55 years. The results showed that menopausal status had a significant effect on cognitive functioning, independent of age. Postmenopausal women showed alterations that are more pronounced in hippocampal activity and connectivity, and poorer performance on a subsequent memory task compared to pre- and perimenopausal women. Endocrine assessments showed that the changes in memory circuitry were related to the loss of ovarian estradiol during menopause.

\section{Attitudes}

Studies also found significant associations between the severity of menopausal symptoms - specifically night sweats, memory loss, and sleeplessness - and negative attitudes toward menopause among postmenopausal women (59). Planned health education related to the climacteric period may be useful in changing women's negative attitudes and beliefs related to menopause. One study showed that the negative beliefs about hot flushes of women who received planned health education decreased, while women who received no education did not change their hot flush beliefs (45).

\section{The prevalence and age of natural menopause}

Two studies assessed the age of natural menopause and factors that are related to earlier onset of natural menopause in highly populated countries such as China (42) and India (60), where premature menopause may affect many women and, consequently, their health. Both studies indicate that socioeconomic and reproductive factors are risk factors for the onset of earlier menopause. Li et al. (42) found 
that the overall median age at natural menopause was 50 years among Chinese women. Lower educational level, poor economic status, lower BMI, age at menarche ( $\leq 14$ years), nulliparity, and smoking were associated with earlier onset of natural menopause. Pallikadavath et al (2016) found that about $8.4 \%$ of women aged $15-49$ years were in natural menopause. A higher risk for natural menopause below 50 years of age was significantly associated with several socioeconomic and reproductive variables: Widowhood or divorce/separation breakdown; poverty; low parity; no schooling; Muslim religion; caste (i.e., lower strata of the caste system); rural residence; Western countries; never using contraceptive pills; not being sterilized; and not having an abortion.

\section{DISCUSSION}

Findings from this study show that socio-demographic predictors were the most common variables related to menopausal symptoms: Specifically education; age; socioeconomic status; family; income; expenditure; economic problems; residence status; marital status; and partner's socio-demographic status (39-45,48-50,52-60). Duration of the reproductive period, higher parity, late perimenopause, and surgical or pharmacological menopause were associated with severe menopausal symptoms (39,40,48,49,52,56-59). A BMI of $\geq 25$ had a significant effect on the severity of menopausal symptoms (psychological, somatic urogenital, and vasomotor) $(39,42,43,47)$. Impairment in physical strength measured by handgrip and memory test showed a significant relation to menopause $(57,58)$. Aerobic exercise for 12-24 weeks had a positive effect on women's muscle strength and was associated with a reduction of menopausal problems (61). Findings also indicate that lifestyle-related factors, such as smoking and coffee intake, were shown to be associated with several menopause-related symptoms, while regular physical activity was inversely associated with menopause-related symptoms $(38-42,44,46)$. Coffee intake (as well as tea and soda) was positively related to a vasomotor symptoms-related problem in postmenopausal women (62). A Spanish study showed that high physical activity could have a positive effect on bone mass (63); moreover, aerobic exercise for 6 months among newly postmenopausal sedentary symptomatic women reduced typical menopausal symptoms - especially night sweats, mood swings, and irritability [62]. These results are supported by the WHO's conclusions that menopause is part of life's various phases and is largely influenced by previous health status, reproductive and sexual health, lifestyle, and the environment.

Prescribing hormone substitute therapy requires careful consideration of benefit and risk and period for hormone intake (1). HT had a beneficial effect on menopause-related symptoms $(36,62)$. To achieve good effects, the exact dosage of hormonal treatment is required, but this area is still being research (16). Hormone replacement therapy (estrogen-progesterone) is associated with increased cancer risk (64) and increased incidence of ischemic and hemorrhagic stroke in the first 6 months after beginning HT (65). The physical activity has a preventive effect against breast cancer but is eliminated by HT therapy (66). Testosterone treatment in postmenopausal women is favorable for sexual health (65) and may improve libido and arousal (66). Estrogen therapy may reduce the risk for depression in later life in menopausal women that are at higher risk for depression (67). Eriksen et al. (2018) conclude that women who start menopausal HT in their 50s have predominantly positive health effects. HT reduced the incidence of diseases such as osteoporosis, colon cancer, and diabetes and had a positive impact on the quality of life (e.g., fewer hot flushes and improved sleep and sexual function) (67). Other studies highlight that non-medical treatment, such as exercise, may offer mental and physical benefits $(68,69)$. In a comparison of 10 -year differences in menopausal status, it was shown that education, smoking, and physical activity play an important role in determining menopausal age. The physical activity was significantly related to a delay in menopause, while smoking was related to an earlier menopausal age (70). In the present study, with regard to reproductive variables related to poorer sexual function, vaginal dryness was the most commonly reported problem $(44,49-51,53,55,59)$. The American Psychological Association has specified that sexual disorders related to menopause are a complex combination of physical and psychological 
disorders impacted by external factors such as an individual's socioeconomic situation, culture, and environment (71). The World Health Organization describes sexual health as a combination of physical, emotional, and social experiences (72). Anxiety and panic disorders (73), as well as poor general health in postmenopausal women, influence sexual health negatively. Vulvovaginal symptoms after menopause impact women's sexual health negatively and are underdiagnosed and undertreated (74).

Study findings also indicate that socio-demographic, socioeconomic, and reproductive factors are risk factors for early menopause for women aged $\leq 50$ years $(42,60)$. Further, poor knowledge of menopause and its related symptoms is linked to negative attitudes, but these were possible to reverse through education (45); moreover, it was shown that postmenopause was significantly associated with higher severity of self-estimated menopause-related symptoms $(40,42-44,47)$. The average duration of menopause-related symptoms, such as anxiety, perceived stress, and depressive symptoms, is approximately 4.5 years following last menstruation (3). Gracez' (2003) study showed that women in generally perceived menopause as part of life but that the majority were aware of the possibility to seek help from medical practitioners for menopausal symptoms (75).

\section{Potential biases}

This study may contain biases. Potential biases of the study may be the analysis of the factors associated with menopausal symptoms, not just the prevalence of symptoms, and the use of standardized instruments for the assessment of menopausal symptoms. Included studies controlled for the effects of age, health history, family history, use of medication, and other relevant factors that might be associated with menopause-related symptoms, apart from the main exposure variables. However, one of the two biases in the studies reviewed is their lack of explanation regarding the inclusion of primary independent variables of interest and possible confounders, and their lack of focus on primary outcomes of interest in a study plan (some studies analyzed a broad range of variables based on data from more extensive studies, without specifying aims that were more focused on the outcomes measured). Furthermore, conclusions related to bone health, cardiovascular health, physical strength and memory changes are limited, due to the dearth of studies that included these outcomes. The use of different types of scales or questionnaires to measure outcomes and the different definitions of the examined constructs may have contributed to inconsistencies in the results. An additional bias may be due to the target population, as there is more data needed on perimenopausal women and the factors associated specifically with the menopausal transition. Only one study included the duration of symptoms, but this study examined individual symptoms and did not provide data on validated scale scores that assess different symptom domains. Most studies measured the severity of symptoms or the impact of menopausal symptoms in the 2 weeks before the investigation, and more studies are needed on the long-term effects of menopause-related changes. Finally, a bias may be that most studies provided cross-sectional data, and thus the direction of the causality between exposure and outcome variables cannot be explored.

\section{CONCLUSIONS}

The research focusing on determinants of menopause-related symptoms in women during the transition to menopause and the postmenopausal period seems to be extensive, with a wide range of variables and instruments having been used. A significant and representative finding was the range of outcomes and independent variables associated with various outcomes from ethnically and geographically diverse populations. In summary, menopause is a consistent physiological condition that can affect the mental, physical, and social dimensions of a woman's life. Some factors linked to her body and the environment in which she lives influences each woman's experience of menopausal symptoms.

\section{COMPETING INTERESTS}

The authors declare that they have no competing interests.

\section{FUNDING}

Thanks to the Faculty of Health Sciences, VID Specialized University, Oslo Norway for financing language review and publication costs. 


\section{REFERENCES}

1. World Health Organization. Research on the Menopause in the 1990s: Report of a WHO Scientific Group. Geneva: World Health Organization; 1996.

2. Burger HG. Physiology and endocrinology of the menopause. J Steroid Biochem Mol Biol 2006;34(1):27-30.

3. Avis NE, Crawford SL, Greendale G, Bromberger JT, Everson-Rose SA, Gold EB, et al. Duration of menopausal vasomotor symptoms over the menopause transition. JAMA Intern Med 2015;175(4):531-9.

https://doi.org/10.1001/jamainternmed.2014.8063.

4. Nappi RE, Particco M, Biglia N, Cagnacci A, Di Carlo C, Luisi S, et al. Attitudes and perceptions towards vulvar and vaginal atrophy in Italian post-menopausal women: Evidence from the European REVIVE survey. Maturitas 2016:91:74-80.

https://doi.org/10.1016/j.maturitas.2016.06.009.

5. Lutz W, Sanderson W, Scherbov SJN. The coming acceleration of global population ageing. Nature 2008:451(7179):716.

https://doi.org/10.1038/nature06516.

6. Lindau ST, Schumm LP, Laumann EO, Levinson W, O'muircheartaigh CA, Waite LJ. A study of sexuality and health among older adults in the United States. N Engl J Med 2007;357(8):762-74.

https://doi.org/10.1056/nejmoa067423.

7. Lochlainn MN, Kenny RA. Sexual activity and aging. Journal of the American Medical Directors Association. 2013;14(8):565-72.

https://doi.org/10.1016/j.jamda.2013.01.022.

8. Minkin MJ. Sexual health and relationships after age 60. Maturitas. 2016;83:27-32.

https://doi.org/10.1016/j.maturitas.2015.10.004

9. Lee DM, Nazroo J, O'Connor DB, Blake M, Pendleton N. Sexual health and well-being among older men and women in England: Findings from the English longitudinal study of ageing. Arch Sex Behav 2016;45(1):133-44. https://doi.org/10.1007/s10508-014-0465-1.

10. World Health Organization. World Health Day 2012: Ageing and Health: Toolkit for Event Organizers. Geneva: World Health Organization; 2012.

11. Ang SB, How CH. Menopause: An important milestone in women's health. Singapore Med J 2013;54(2):60-3.

12. Thomas HN, Thurston RC. A biopsychosocial approach to women's sexual function and dysfunction at midlife: A narrative review. Maturitas 2016;87:49-60.

https://doi.org/10.1016/j.maturitas.2016.02.009.

13. Ghorbani R, Nassaji M, Shahbazi A, Tabar SB, Rahaei F. Attitudes toward menopause among middle-aged women in Semnan, Iran. J Egypt Public Health Assoc 2014;89(1):42-5.

https://doi.org/10.1097/01.epx.0000443986.36638.73.

14. Montemurro B, Siefken JM. Cougars on the prowl? New perceptions of older women's sexuality. J Aging Stud 2014;28:35-43.

https://doi.org/10.1016/j.jaging.2013.11.004

15. Sayakhot $P$, Vincent A, Teede H. Cross-cultural study: Experience, understanding of menopause, and related therapies in Australian and Laotian women. Menopause 2012;19(12):1300-8. https://doi.org/10.1097/gme.0b013e31825fd14e.

16. Stevenson JC. HRT and cardiovascular disease. Best Pract Res Clin Obstet Gynaecol 2009;23(1):109-20.

17. Valadares AL, Machado VS, da Costa-Paiva LS, de Souza MH, Osis MJ, Pinto-Neto AM. Sexual activity in Brazilian women aged 50 years or older within the framework of a population-based study. Menopause 2014;21(3):295-300

https://doi.org/10.1097/gme.0b013e3182987231.

18. Castellanos-Torres E, Álvarez-Dardet C, Ruiz-Muñoz D, Pérez G. Social determinants of sexual satisfaction in Spain considered from the gender perspective. Ann Epidemiol 2013;23(3):150-6.

https://doi.org/10.1016/j.annepidem.2012.12.010.

19. Brody S. Obstetrik och Gynekologi: Medicinsk Grundutbildning; 1970.

20. Kaplan A, Hogg B, Hildingsson I, Lundgren I. Lärobok för Barnmorskor. Lund, Sweden: Studentlitteratur; 2009.

21. Hoga L, Rodolpho J, Gonçalves B, Quirino B. Women's experience of menopause: A systematic review of qualitative evidence. JBI Database Syst Rev Implement Rep 2015;13(8):250-337. https://doi. org/10.11124/01938924-201513080-00018

22. Chou MF, Wun YT, Pang SM. Menopausal symptoms and the menopausa rating scale among midlife Chinese women in Macau, China. Women Health 2014;54(2):115-26. https://doi.org/10.1080/03630242.2013.871767.

23. Barlow DH, Cardozo LD, Francis RM, Griffin M, Hart DM, Stephens E, et al. Urogenital ageing and its effect on sexual health in older British women. BJOG Int J Obstet Gynaecol 1997;104(1):87-91.

https://doi.org/10.1111/j.1471-0528.1997.tb10655.x.

24. Society NA. Symptomatic vulvovaginal atrophy advisory panel. Management of symptomatic vulvovaginal atrophy: 2013 position statement of the North American Menopause society. Menopause 2013;20(9):888-902.

https://doi.org/10.1097/gme.0b013e3182a122c2.

25. Hughes AK, Rostant OS, Pelon S. Sexual problems among older women by age and race. J Womens Health 2015;24(8):663-9.

https://doi.org/10.1089/jwh.2014.5010

26. Cabral PU, Canário AC, Spyrides MH, Uchôa SA, Eleutério JJ, Giraldo PC et al. Physical activity and sexual function in middle-aged women. Rev Assoc Méd Bras 2014;60(1):47-52.

https://doi.org/10.1590/1806-9282.60.01.011

27. Sanchez-Borrego R, Molero F, Castano R, Castelo-Branco C, Honrado M, Jurado AR, et al. Spanish consensus on sexual health in men and women over 50. Maturitas 2014;78(2):138-45.

https://doi.org/10.1016/j.maturitas.2014.02.020.

28. Yang CF, Kenney NJ, Chang TC, Chang SR. Sex life and role identity in Taiwanese women during menopause: A qualitative study. J Adv Nurs 2016;72(4):770-81.

https://doi.org/10.1111/jan.12866.

29. Robak-Cholubek D, Wdowiak A, Makara-Studzinska M, Korczynska E. Perception and degree of acceptance of menopause-related changes in various spheres of life by postmenopausal women. Ann Agric Environ Med 2014;21(3):666-9. https://doi.org/10.5604/12321966.1120622.

30. Merghati-Khoei E, Sheikhan F, Shamsalizadeh N, Haghani H, Yousofnia Pasha YR, Killeen T. Menopause negatively impacts sexual lives of middle-aged Iranian women: A cross-sectional study. J Sex Marital Ther 2014;40(6):552-60. https://doi.org/10.1080/0092623x.2013.796577

31. Mitchell KR, Mercer CH, Ploubidis GB, Jones KG, Datta J, Field N, et al. Sexual function in Britain: Findings from the third national survey of sexual attitudes and lifestyles (Natsal-3). Lancet 2013;382(9907):1817-29.

https://doi.org/10.1016/s0140-6736(13)62366-1.

32. Worsley R, Bell RJ, Gartoulla P, Davis SR. Low use of effective and safe therapies for moderate to severe menopausal symptoms: A cross-sectional community study of Australian women. Menopause 2016;23(1):11-7. https://doi.org/10.1097/gme.0000000000000495.

33. Liberati A, Altman DG, Tetzlaff J, Mulrow C, Gøtzsche PC, loannidis JP, et al. The PRISMA statement for reporting systematic reviews and meta-analyses of studies that evaluate health care interventions: Explanation and elaboration. PLoS Med. 2009;6(7):e1000100. 
https://doi.org/10.1371/journal.pmed.1000100.

34. Babineau J. Product review: Covidence (systematic review software). J Can Health Lib Assoc 2014;35(2):68-71.

https://doi.org/10.5596/c14-016.

35. Norwegian Institute of Public Health. Sjekklister for Vurdering av Forskningsartikler; 2017. Available from: https://www.fhi. no/kk/oppsummert-forskning-for-helsetjenesten/sjekklister-for -vurdering-av-forskningsartikler.

36. Boyle MH. Guidelines for evaluating prevalence studies. Evid Based Ment Health. 1998;1(2):37-9.

37. Guyatt G, Rennie D, Meade M, Cook D. Users' Guides to the Medical Literature. New York: McGraw-Hill Medical; 2015.

38. Singh J. Critical appraisal skills programme. J Pharmacol Pharmacother 2013;4(1):76.

39. Alquaiz J, Siddiqui A, Tayel S, Habib F. Determinants of severity of menopausal symptoms among Saudi women in Riyadh city. Climacteric 2014;17(1):71-8.

https://doi.org/10.3109/13697137.2013.815161.

40. Gartoulla P, Worsley R, Bell RJ, Davis SR. Moderate to severe vasomotor and sexual symptoms remain problematic for women aged 60 to 65 years. Menopause 2015;22(7):694-701.

https://doi.org/10.1097/gme.0000000000000383.

41. Javadivala Z, Kousha A, Allahverdipour $\mathrm{H}$, Jafarabadi MA, Tallebian $\mathrm{H}$. Modeling the relationship between physical activity and quality of life in menopausal-aged women: A cross-sectional study. J Res Health Sci 2013;13(2):168-75.

42. Li L, Wu J, Pu D, Zhao Y, Wan C, Sun L, et al. Factors associated with the age of natural menopause and menopausal symptoms in Chinese women. Maturitas 2012;73(4):354-60.

https://doi.org/10.1016/j.maturitas.2012.09.008.

43. Ornat L, Martínez-Dearth R, Muñoz A, Franco P, Alonso B, Tajada M, et al. Sexual function, satisfaction with life and menopausal symptoms in middle-aged women. Maturitas 2013;75(3):261-9.

https://doi.org/10.1016/j.maturitas.2013.04.007.

44. Prairie BA, Wisniewski SR, Luther J, Hess R, Thurston RC, Wisner KL, et al. Symptoms of depressed mood, disturbed sleep, and sexual problems in midlife women: Cross-sectional data from the study of women's health across the nation. J Womens Health 2015;24(2):119-26.

https://doi.org/10.1089/jwh.2014.4798.

45. Çelik AS, Pasinlioğlu T. Effects of imparting planned health education on hot flush beliefs and quality of life of climacteric women. Climacteric 2017;20(1):25-30.

https://doi.org/10.1080/13697137.2016.1249839.

46. Som N, Roy P, Ray SJC. Menopause-specific quality of life of a group of urban women, West Bengal, India. Climacteric 2014;17(6):713-9. https://doi.org/10.3109/13697137.2014.913283.

47. Yim G, Ahn Y, Chang Y, Ryu S, Lim JY, Kang D, et al. Prevalence and severity of menopause symptoms and associated factors across menopause status in Korean women. Menopause 2015;22(10):1108-16.

https://doi.org/10.1097/gme.0000000000000438.

48. Cabral PU, Canário AC, Spyrides MH, Uchôa SA, Eleutério J, Gonçalves AK. Determinants of sexual dysfunction among middlelaged women. Int J Gynecol Obstet 2013;120(3):271-4.

https://doi.org/10.1016/j.jiggo.2012.09.023.

49. Field N, Mercer CH, Sonnenberg P, Tanton C, Clifton S, Mitchell KR, et al. Associations between health and sexual lifestyles in Britain: Findings from the third national survey of sexual attitudes and lifestyles (Natsal-3). Lancet 2013;382(9907):1830-44.

https://doi.org/10.1016/s0140-6736(13)62222-9.
50. Jonusiene G, Zilaitiene B, Adomaitiene V, Aniuliene R, Bancroft JJC. Sexual function, mood and menopause symptoms in Lithuanian postmenopausal women. Climacteric 2012;16(1):185-93.

https://doi.org/10.3109/13697137.2012.682746.

51. Tungmunsakulchai R, Chaikittisilpa S, Snabboon T, Panyakhamlerd $\mathrm{K}$, Jaisamrarn U, Taechakraichana NJ. Effectiveness of a low dose testosterone undecanoate to improve sexual function in postmenopausal women. BMC Womens Health 2015;15(1):113.

https://doi.org/10.1186/s12905-015-0270-6.

52. Hunter MM, Nakagawa S, Van Den Eeden SK, Kuppermann M, Huang AJ. Predictors of impact of vaginal symptoms in postmenopausal women. Menopause (New York, NY) 2016;23(1):40. https://doi.org/10.1097/gme.0000000000000482.

53. Jaspers L, Dhana K, Muka T, Meun C, Kiefte-de Jong JC, Hofman A, et al. Sex steroids, sex hormone-binding globulin and cardiovascular health in men and postmenopausal women: The Rotterdam study. J Clin Endocrinol Metab 2016;101(7):2844-52.

https://doi.org/10.1210/jc.2016-1435.

54. Kutob RM, Yuan NP, Wertheim BC, Sbarra DA, Loucks EB, Nassir R, et al. Relationship between marital transitions, health behaviors, and health indicators of postmenopausal women: Results from the women's health initiative. J Womens Health 2017;26(4):313-20.

https://doi.org/10.1089/jwh.2016.5925

55. Lo S. Bone health status of postmenopausal Chinese women. Hong Kong Med J 2015;21(6):536-41.

56. DiBonaventura M, Luo X, Moffatt M, Bushmakin AG, Kumar M, Bobula J. The association between vulvovaginal atrophy symptoms and quality of life among postmenopausal women in the United States and Western Europe. J Womens Health 2015;24(9):713-22.

https://doi.org/10.1089/jwh.2014.5177.

57. Jacobs EG, Weiss BK, Makris N, Whitfield-Gabrieli S, Buka SL, Klibanski A et al. Impact of sex and menopausal status on episodic memory circuitry in early midlife. J Neurosci 2016;36(39):10163-73.

https://doi.org/10.1523/jneurosci.0951-16.2016.

58. da Câmara SM, Zunzunegui MV, Pirkle C, Moreira MA, Maciel ÁC. Menopausal status and physical performance in middle aged women: A cross-sectional community-based study in northeast Brazil. PLoS One 2015;10(3):e0119480

https://doi.org/10.1371/journal.pone.0119480.

59. Ghazanfarpour M, Kaviani M, Abdolahian S, Bonakchi H, Khadijeh MN, Naghavi M, et al. The relationship between women's attitude towards menopause and menopausal symptoms among postmenopausal women. Gynecol Endocrinol 2015;31(11):860-5.

https://doi.org/10.3109/09513590.2015.1056138.

60. Pallikadavath S, Ogollah R, Singh A, Dean T, Dewey A, Stones W. Natural menopause among women below 50 years in India: A population-based study. Indian J Med Res 2016;144(3):366.

https://doi.org/10.4103/0971-5916.198676

61. Karacan SJ. Effects of long-term aerobic exercise on physical fitness and postmenopausal symptoms with menopausal rating scale. Sci Sports 2010;25(1):39-46.

https://doi.org/10.1016/j.scispo.2009.07.004.

62. Faubion SS, Sood R, Thielen JM, Shuster LT. Caffeine and menopausal symptoms: What is the association? Menopause 2015;22(2):155-8.

https://doi.org/10.1097/gme.0000000000000301.

63. Calderon-Garcia JF, Lavado-Garcia JM, Martin RR, Moran JM, CanalMacias ML, Pedrera-Zamorano JD. Bone ultrasound and physical activity in postmenopausal Spanish women. Biol Res Nurs 2013;15(4):416-21.

https://doi.org/10.1177/1099800412459800. 
64. Beral V; Million Women Study Collaborators. Breast cancer and hormone-replacement therapy in the Million Women Study. Lancet 2003;362(9382):419-27.

https://doi.org/10.1016/s0140-6736(03)14065-2.

65. Lemaitre RN, Heckbert SR, Psaty BM, Smith NL, Kaplan RC, Longstreth WJ. Hormone replacement therapy and associated risk of stroke in postmenopausal women. Arch Intern Med 2002;162(17):1954-60. https://doi.org/10.1001/archinte.162.17.1954.

66. Pizot C, Boniol M, Mullie P, Koechlin A, Boniol M, Boyle P, et al. Physical activity, hormone replacement therapy and breast cancer risk: A meta-analysis of prospective studies. Eur J Cancer 2016;52:138-54.

https://doi.org/10.1016/j.ejca.2015.10.063.

67. Eriksen $\mathrm{E}$, Moen $\mathrm{M}$, Iversen OJ. Østrogener i menopausen på tide å endre anbefalingene? Tidsskr Nor Legeforen 2018;138(6).

https://doi.org/10.4045/tidsskr.17.1059.

68. Daley A, Stokes-Lampard H, Thomas A, MacArthur CJ. Exercise for vasomotor menopausal symptoms. Cochrane Database Syst Rev 2014;11:CD006108.

https://doi.org/10.1002/14651858.cd006108.pub4.

69. Daley AJ, Thomas A, Roalfe AK, Stokes-Lampard H, Coleman S, Rees M, et al. The effectiveness of exercise as treatment for vasomotor menopausal symptoms: Randomised controlled trial. BJOG 2015;122(4):565-75. https://doi.org/10.1111/1471-0528.13193.
70. Pakarinen M, Raitanen J, Kaaja R, Luoto RJ. Secular trend in the menopausal age in Finland 1997-2007 and correlation with socioeconomic, reproductive and lifestyle factors. Maturitas 2010;66(4):417-22.

https://doi.org/10.1016/j.maturitas.2010.04.005.

71. American Psychiatric. Diagnostic and Statistical Manual of Mental Disorders (DSM-5®): American Psychiatric Publication; 2013.

72. World Health Organization. Defining Sexual Health: Report of a Technical Consultation on Sexual Health, 28-31 January 2002, Geneva: World Health Organization; 2006.

https://doi.org/10.1016/s0968-8080(02)00085-x.

73. Dèttore D, Pucciarelli M, Santarnecchi EJ. Anxiety and female sexual functioning: An empirical study. J Sex Marital Ther 2013;39(3):216-40.

https://doi.org/10.1080/0092623x.2011.606879.

74. Simon JA, Nappi RE, Kingsberg SA, Maamari R, Brown V. Clarifying vaginal atrophy's impact on sex and relationships (CLOSER) survey: Emotional and physical impact of vaginal discomfort on North American postmenopausal women and their partners. Menopause 2014;21(2):137-42.

https://doi.org/10.1097/gme.0b013e318295236f.

75. Gracez T. Older Women's Experiences of Menopause: A Feminist Descriptive Study of Older Women's Knowledge and Attitudes toward Menopause: A Thesis Presented in Partial Fulfillment of the Requirements for the Degree of Master of Arts in Nursing, Massey University. Palmerston North: Massey University; 2003. 